\title{
Contribution of type W human endogenous retroviruses to the human genome: characterization of HERV-W proviral insertions and processed pseudogenes
}

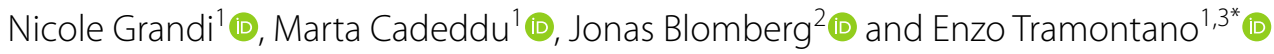

\begin{abstract}
Background: Human endogenous retroviruses (HERVs) are ancient sequences integrated in the germ line cells and vertically transmitted through the offspring constituting about $8 \%$ of our genome. In time, HERVs accumulated mutations that compromised their coding capacity. A prominent exception is HERV-W locus 7q21.2, producing a functional Env protein (Syncytin-1) coopted for placental syncytiotrophoblast formation. While expression of HERV-W sequences has been investigated for their correlation to disease, an exhaustive description of the group composition and characteristics is still not available and current HERV-W group information derive from studies published a few years ago that, of course, used the rough assemblies of the human genome available at that time. This hampers the comparison and correlation with current human genome assemblies.

Results: In the present work we identified and described in detail the distribution and genetic composition of 213 HERV-W elements. The bioinformatics analysis led to the characterization of several previously unreported features and provided a phylogenetic classification of two main subgroups with different age and structural characteristics. New facts on HERV-W genomic context of insertion and co-localization with sequences putatively involved in disease development are also reported.

Conclusions: The present work is a detailed overview of the HERV-W contribution to the human genome and provides a robust genetic background useful to clarify HERV-W role in pathologies with poorly understood etiology, representing, to our knowledge, the most complete and exhaustive HERV-W dataset up to date.
\end{abstract}

Keywords: HERV-W, Endogenous retroviruses, HERV, Syncytin

\section{Background}

More than 40 years after the first evidence of discrepancy between the amount of genetic material and organisms complexity, it is now established that less than $2 \%$ of the human genome is composed of protein-coding regions [1]. With respect to this data, it is impressive to consider that human endogenous retroviruses (HERVs) represent four times this value, constituting about the $8 \%$ of our

\footnotetext{
*Correspondence: tramon@unica.it

${ }^{1}$ Department of Life and Environmental Sciences, University of Cagliari, Cittadella Universitaria di Monserrato SS554, 09042 Monserrato, Cagliari, Italy

Full list of author information is available at the end of the article
}

DNA [2]. HERV sequences seem to have been acquired through a traditional infective process, occurred mostly over 30 million years ago [3]. The reverse transcription of the viral genome and the further integration into the germ line cells allowed the Mendelian transmission of these elements through the offspring, determining their coevolution with the host genome.

HERVs belong to class-I transposable elements, termed also retrotransposons, which duplicate through a reversetranscribed RNA intermediate. Beside HERVs this group comprises also elements devoid of long terminal repeats (LTRs), such as long and short interspersed nuclear elements (LINEs and SINEs respectively). Despite their 
abundant presence, HERV classification has been for a long time incomplete and sometimes controversial [4], and a comprehensive dataset of the HERV elements present in the human genome has been only recently provided [5]. In particular, HERVs are distributed among three main classes based on sequence similarity with the exogenous members: class I (Gammaretrovirus- and Epsilonretrovirus-like), class II (Betaretrovirus-like) and class III (Spumaretrovirus-like). Each class encloses a variable number of groups [5]. HERV groups have been traditionally identified with a letter according to the type of human tRNA that binds the primer binding site (PBS) during the reverse transcription process [6]. For example, HERV-K elements are supposed to use a Lysine tRNA. Some groups have also been occasionally named according to a neighbor gene (HERV-ADP) or a particular amino acid motif (HERV-FRD). These nomenclatures are now considered inadequate, and taxonomic studies of HERV groups are usually performed using a phylogenetic approach, commonly based on the highly conserved pol gene [7]. Currently, HERV primary integrations can be divided into 39 groups, and this panorama is further complicated by 31 additional "non canonical" groups of mosaic forms arisen from secondary integrations or recombination events [5].

For few HERV groups, viral spreading in human chromosomes was not only due to new infections generating novel provirus integrations, but it was also mediated by alternative mechanisms. It is in fact known that several elements of the HERV-W multi-copy group derive from the retrotranscription and mobilization of proviral RNA transcripts mediated by human LINE (L1) machinery, that is responsible for their insertion into new genomic regions. Those sequences are structurally colinear with the proviral mRNA and are called processed pseudogenes [8]. Moreover, the human genome harbors several hundreds of solitary HERV-W LTRs deriving from homologous recombination between the $5^{\prime}$ - and $3^{\prime}$ LTRs that removed the retroviral internal part [9].

Regardless of the mechanism of formation, the genomic persistence of HERV sequences during evolution led to the accumulation of several mutations, insertions and deletions, that have generally compromised their coding capacity [10]. A prominent exception is once again represented by the HERV-W group. Initially identified for its possible role in Multiple Sclerosis (MS), this group showed a high expression level in placental tissues. Further investigations interestingly revealed that an HERV-W provirus, named ERVWE1 and localized to locus 7q21.2, (1) retained a complete env Open Reading Frame (ORF) [11]; (2) was able to produce a functional protein, called Syncytin-1 and (3) had been co-opted by the human genome for the trophoblast cells fusion during pregnancy, an important structure for regulating the exchanges between mother and fetus [12-14].

Starting from these findings, the expression and coding capacity of HERV-W group have been investigated in the different tissues, above all to find a correlation to various diseases, such as MS [15-21], Schizophrenia [22, 23] and bipolar disorder [24,25], comprising also a number of pathologies with poorly understood etiology, such as osteoarthritis and cutaneous $\mathrm{T}$ cell lymphoma [26, 27]. However, despite the great interest in HERV-W expression, no definitive correlation with human pathologies have been conclusively demonstrated so far [28] and the characterization of the group at the genomic level still remains a major genetic goal and a bioinformatics challenge [29]. Specifically, the current knowledge of the HERV-W genomic distribution and number of copies is still referred to analyses performed a few years ago [8, 30, 31]. In particular, Voisset et al. described the presence of 70, 100, and 30 HERV-Wrelated gag, pro, and env regions respectively, using a PCR approach on isolated chromosomes DNA samples with HERV-W-specific primers [30]. Costas identified a total of 140 HERV-W elements through a NCBI BLAST within the draft sequence of the human genome [31]. Pavlícek et al. reported 311 HERV-W elements and 343 solitary LTRs identified using the RepeatMasker program in the GoldenPath assembly of $87 \%$ of the human genome [8] These works represent milestones in the HERV-W group characterization, but the absence of a complete and exhaustive version of the human genome and the use of different methodologies make it hard to compare and correlate these data with the current version of the human genome.

Moreover, with the exception of the well-described Syncytin-1 provirus, detailed information about the group composition and its members characteristics are somehow lacking, preventing a comprehensive analysis of their possible involvement in human pathologies. In fact, a detailed knowledge of HERV-W genic origin is essential to complete the previously mentioned observed expression profiles [16-25, 32] and to evaluate their possible involvement in disease development and/or progression. Furthermore, it is well known that the mere presence of HERV integrated elements could affect human physiology and health through alternative mechanisms even in the absence of gene expression or products. This can occur for example (1) with gene physical disruption after HERV insertion [33, 34]; (2) by damaging recombination events that can produce genomic alterations ranging from deletions and duplications to large-scale chromosomal rearrangements [35]; and (3) through the effects exerted by HERVs and their LTRs that naturally present promoters, enhancers, polyadenylation signals and splice donor sites [5, 36-38] and can regulate also human genes expression in a tissue specific manner [39-49]. 
In this context, the current HERV-W expression studies seem to be not exhaustive to understand the real effects that these elements can exert. In fact, on the one side, due to their multi-copy nature, it is not always clear from which genomic locus a HERV-W mRNA is transcribed, and, on the other side, the potential effects of such sequences is not solely connected to their expression capacity, but depends also on their localization and their ability to (dys)regulate host functions also through alternative mechanisms behind the presence of a RNA/protein products.

In the light of this, the definition of a precise and updated HERV-W genomic map is a pressing need to better evaluate their role in human health and their real influence on host genome. Here we report a comprehensive analysis of HERV-W sequences presence and distribution within human genome, with a detailed description of the different structural and phylogenetic aspects characterizing the group.

\section{Results}

\section{HERV-W identification and general classification}

In a recent work aimed to the global classification of HERV clades and sequences in the human genome, we reported the presence of 126 elements belonging to HERV-W group [5]. These data were obtained through the bioinformatics tool RetroTector (ReTe), a program package implemented for the identification of ERV full integrations in vertebrate genomes and the attempted reconstruction of the relative ORFs and proteins [50]. For HERV sequences recognition ReTe uses a collection of generic, conserved motifs, a few within env and gag genes, that can be mutated or lost in defective proviruses [5]. Such "bias" was reported as responsible for the low representation of HERV Class III proviruses that have an aberrant gag and may not have an env [5]. In the light of this, willing to build an updated dataset of HERV-W sequences in the human genome GRCh37/hg19 assembly, we used a combined strategy based on (1) the ReTe analysis and (2) a traditional Genome Browser BLAT search [51], using the assembled RepBase reference LTR17-HERV17-LTR17 [52] as a query. This integrated approach led to the characterization of a total of 213 HERV-W related sequences: the 126 previously identified by ReTe and 87 additional elements retrieved by Genome Browser BLAT. Indeed, a high proportion of newly identified HERV-W sequences showed huge and recurrent deletions that caused loss of extended portions in gag, pol and $e n v$ genes (described more in detail in the structural characterization section). Hence, the defective nature of the great majority of HERV-W sequences could be responsible for the underrepresentation of the ReTe outcome, confirming the importance of a double approach in HERV identification.
The main characteristics of HERV-W elements are summarized in supplementary material (Additional file 1: Table S1). We named the HERV-W elements according to their genomic localization, in order to have a unique and direct identification of each sequence. In the presence of multiple sequences in the same locus, the order within the band is expressed with a letter following the alphabetical order as previously described [53]. HERVW elements occurred on all chromosomes showing no recognizable cluster distribution, except chromosome 16 that apparently do not contain HERV-W proviruses or pseudogenes.

The 213 HERV-W sequences were firstly divided into three categories due to previously reported structural characteristics that mostly address the LTRs portion and that reflect their mechanism of formation [8]: proviruses (65), processed pseudogenes (135) and undefined elements (13). Briefly, with respect to the LTR17 RepBase consensus (780 nucleotides), proviral sequences show complete LTRs (referred here as proviral LTRs) and have been inserted into human DNA by a traditional process of retroviral integration. Proviral LTRs show a traditional composition with two unique regions (U3 and U5) separated by a repeated portion (R), giving a U3-R-U5 structure. As described by Pavlícek et al. [8], pseudogenes are LINE-1 processed HERV-W sequences presenting (1) truncated LTRs (referred here as pseudogenic LTRs), with the $5^{\prime}$ LTR showing a R-U5 structure (start from nucleotide 256 of the consensus) and the $3^{\prime}$ LTR showing a U3-R structure (end at position 326 of the consensus), (2) a poly(A) tail of variable length, and (3) a common TT/AAAA insertion motif and a variablelength (5-15 bp) target site duplication [8]. Finally, undefined elements are sequences that have lost those regions in both LTRs and so remained undefined due to the absence of the signatures described above.

It is interesting to note that our results differed from previous analysis performed a number of years ago on not exhaustive draft versions of the human genome [8, $30,31]$ and with the use of different detecting methodologies, leading to discordant results that are not always easy to retrieve and correlate with current data. In fact, on one side, two studies on HERV-W distribution and composition [30,31] reported a lower number of elements with respect to our dataset. In particular, Voisset et al. described the presence of 70, 100, and 30 HERVW-related gag, pro, and env regions, respectively, without further indications about their origin [30], while Costas identified a total of 140 HERV-W elements, 73 less than the present analysis. On the other side, when compared to our dataset, the study by Pavlicek et al. reported a higher number of HERV-W sequences (311) [8]. The lack of available supplementary information of Pavlicek 
HERV-W dataset (e.g. nucleotide sequences or genomic localization) did not allow us to perform a direct comparison with our results. However, Pavlicek et al. HERVW sequences were retrieved from a draft version of the human genome using the RepeatMasker program that, in the presence of the recurrent and huge deletions such as the ones observed in the HERV-W sequences, could not easily identify the whole elements. Hence, more fragments previously reported as independent elements possibly belonged to the same provirus/pseudogene. This hypothesis seems to be confirmed by a subsequent study where the same dataset has been used for the HERV-W processed pseudogenes length distribution analysis [54]. Such report showed that the most represented length class in Pavlicek dataset enclosed very short sequences $(0-0.5 \mathrm{~Kb})$, with a low proportion of $>3.5 \mathrm{~Kb}$ elements. Differently, in our dataset $>90 \%$ of sequences are in the $1-7.5 \mathrm{~Kb}$ range, with around $25 \%>6.5 \mathrm{~Kb}$. Overall, the use of the Rete software, that relates retroviral elements reconstructing the original chain [50], together with a visual inspection of all aligned sequences plus their flanking sites of integration with respect to the group reference, probably led to more reliable sequence recognition. Furthermore, the overestimation of HERV-W members in Pavlicek dataset could also be due to the possible inclusion of HERV9 sequences, highly related to HERV-W but constituting a separate phylogenetic group [5]. In fact, to avoid such bias we initially included a HERV9 consensus in every HERV-W phylogenetic trees, assuring that none of the sequences classified as HERV-W clustered with HERV9 group (data not shown). Importantly, a significant contribution on the HERV-W group presence in the human genome was recently provided in a study in which the cDNA obtained from HERV-W RNA transcripts in MS patients and controls brain samples was amplified in the env region and assigned to single HERV-W loci by Genome Browser BLAT on the NCBI36/hg18 assembly (March 2006) [20]. While the purpose of that study was not a HERV-W group genomic characterization and was biased for env sequences analysis, yet it provided a remarkable genomic map of 176 HERV-W loci, enclosing 35 proviruses, in their supplementary material [20]. Noteworthy, with respect to this study, our analysis led to the identification of 37 further HERV-W elements (9 proviruses, 18 processed pseudogenes and 10 undefined sequences), and to a more defined classification of proviruses and processed pseudogenes.

\section{Structural characterization}

In order to characterize the HERV-W structure we firstly aligned and analyzed the 213 sequences dataset with respect to the assembled reference
LTR17-HERV17-LTR17, built from RepBase Update consensus sequences for HERV-W LTRs and internal portion [52]. HERV-W sequences showed a typical proviral structure, with the gag, pro, pol and env genes flanked by two LTRs. Briefly, the gag gene (nucleotides 2718-4191) encodes the structural components of matrix (MA), capsid (CA) and nucleocapsid (NC); the pro-pol genes (4195-7692) determine the production of the three viral enzymes Protease, Reverse Transcriptase (RT) and Integrase (IN); and the env gene (7720-9348) is responsible for encoding the envelope surface (SU) and transmembrane (TM) elements. The $5^{\prime}$ - and $3^{\prime}$ LTRs (1-780 and 9406-10186, respectively) are formed during the retrotranscription process and are identical at time of integration. In addition, almost all HERV-W identified sequences present a $2 \mathrm{~Kb}$ long non-coding region, located between $5^{\prime}$ LTR and gag gene and characterized by an AG rich expansion of variable length. This portion was previously reported for three cDNA HERV-W clones [11], but neither function or origin has been proposed or demonstrated yet.

Firstly, comparing proviral versus pseudogenic sequences we asked whether, beside the shorter pseudogenic LTRs, the internal sequence completeness was comparable. We evaluated the presence of each retroviral element (5' LTR, gag, pro, pol, env and $3^{\prime}$ LTR) in the three classes of HERV-W sequences (proviruses, processed pseudogenes and undefined), considering as retained all elements with at least the $20 \%$ of nucleotides with respect to the correspondent element in LTR17 and HERV17 RepBase consensus (Fig. 1). Analysis of all identified HERV-Ws showed that proviral sequences appear to be the most complete, with a better general maintenance of the considered retroviral structures, while pseudogenes, interestingly, frequently lack the $5^{\prime}$ LTR, absent in $>50 \%$ of elements, and gag and pro genes (Fig. 1). Importantly, for all classes, env is the most frequently lost element, due to recurrent extended deletions that involve $>80 \%$ of the gene. In addition, besides the lack of both LTRs, undefined sequences showed a higher frequency of gene loss, especially in the pro-pol-env portions, indicating that deletion processes were not limited to the LTR sequences.

Secondly, in order to define the group structural characteristics, the 213 HERV-W elements were further analyzed in great detail by annotating all insertions/deletions with respect to the consensus LTR17-HERV17-LTR17, as schematically represented for the 59 proviruses with minimum length of $2.5 \mathrm{~Kb}$ in Fig. 2. In comparison to the consensus, in all types of sequences some recurrent deletions clearly affect viral genes, with the loss of some big viral portions: (1) nucleotides 2780-3209 in gag gene (45\% of the sequences), (2) nucleotides $4513-6184$ and 


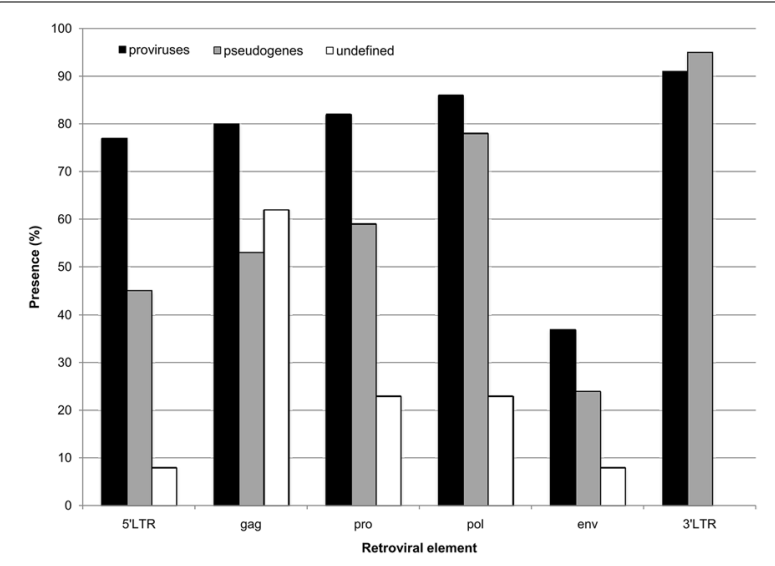

Fig. 1 Overview on the HERV-W structural completeness. The presence of each retroviral element in the three classes of HERV-W sequences is depicted. An element is considered as retained if at least the $20 \%$ of the sequence is present (lengths are referred to LTR17HERV17 reference elements). With respect to the LTR17 RepBase consensus (780 nucleotides), proviral sequences show complete LTRs, while processed pseudogenes present typically truncated LTRs due to the L1-mediated retroposition, lacking U3 in 5' LTR (R-U5 structure, nucleotides 256-780) and U5 in 3' LTR (U3-R structure, nucleotides $1-326)$

$6797-7692$ (IN portion) in pol gene ( 28 and $84 \%$ of the sequences, respectively), and (3) nucleotides 7928-9114 in the env gene ( $85 \%$ of the sequences), with the exception of a small region of about 30 nucleotides at position 8289-8318 that is frequently present despite the flanking deletions. Interestingly, the recurrent loss of pol and env genes, deleted in the $\mathrm{C}$-terminal IN portion and retaining only the TM intracytoplasmic tail, respectively, possibly suggests a selective removal of regions that were no longer needed in the absence of an active infective transmission.

In addition to these major mutations, the analyses highlighted a greater amount of minor insertions/deletions and single nucleotides substitutions that, overall, allow to specifically identify the uniqueness of each HERW-W sequence. The majority of these variations appear to be randomly distributed among the sequences, as expected from the normal random genomic substitution rate, while a number of them are shared by the great majority of the sequences and characterize their structure with respect to the reference. This analysis allowed also to better defining a new HERV-W consensus generated from our proviral dataset that we graphically compared with the LTR17-HERV17-LTR17 consensus (Fig. 3). Interestingly, the LTR structures of the new HERV-W consensus showed recurrent mutations defining two subgroups of sequences that were used, in combination with the phylogenetic analysis, as key positions for subgroup definition.

\section{Phylogenetic analysis and HERV-W proposed subgroup classification}

In order to clarify the phylogenetic and evolutionary relationship within the group, LTRs and viral genes were analyzed through the construction of phylogenetic trees using both a neighbor-joining (NJ) method (Fig. 4; Additional file 2: Fig. S1) and a maximum likelihood (ML) analysis (data not shown). Both analysis yielded similar trees. In addition, since HERV9 sequences have been reported to be highly related to HERV-W, to exclude possible misclassifications, a HERV9 generated consensus [5] was initially included in all trees in order to identify any member of this HERV-W related family that could be misinterpreted during the sequence collection. As expected, the HERV9 consensus was clearly separated from the HERV-W sequences, which grouped together showing a $100 \%$ bootstrap value in every tree (data not shown).

In the case of proviral sequences, the $5^{\prime}$ - and $3^{\prime}$ LTRs were analyzed together in the same phylogenetic tree (Fig. 4a). On the contrary, the truncated structure of pseudogenic $5^{\prime}$ - and $3^{\prime}$ LTRs only yields a short common region ( $\mathrm{R}$; about 90 nucleotides) necessitating a separate analysis (Fig. 4b, c). gag, pol and env genes trees are included in supplementary material in Additional file 2: Fig. S1.

\section{LTRs}

In LTR trees, the distribution of proviral and pseudogenic sequences in two major clusters allowed us to divide them into two distinct subgroups, named 1 and 2. The subgroup of HERV-W single members is reported in Additional file 1: Table S1. Within the 213 HERV-W group members, $69 \%$ of the sequences belong to subgroup 1 (38 proviruses and 108 pseudogenes), while $24 \%$ of them belonged to subgroup 2 (25 proviruses and 27 pseudogenes). The remaining $7 \%$ was constituted of sequences lacking both LTRs and that, subsequently, could not be classified.

Considering that the subgroup division was generally not well supported by bootstrap values, < $50 \%$ except for pseudogenic $3^{\prime}$ LTR (90\%), the identified HERV-W clusters were further analyzed using common features. The members of each subgroup were aligned and compared with respect to LTR17 reference in order to find characteristic features that could confirm and support the robustness of the classification. In general, subgroup 1 elements were not characterized by significant mutations with respect to the reference sequence, probably because LTR17 and HERV17 consensus were built from a few of these elements, such as the 7q21.2 Syncytin-1 locus. A pairwise distance calculation confirmed that the average identity with LTR17 was around $93 \%$. Contrarily, 


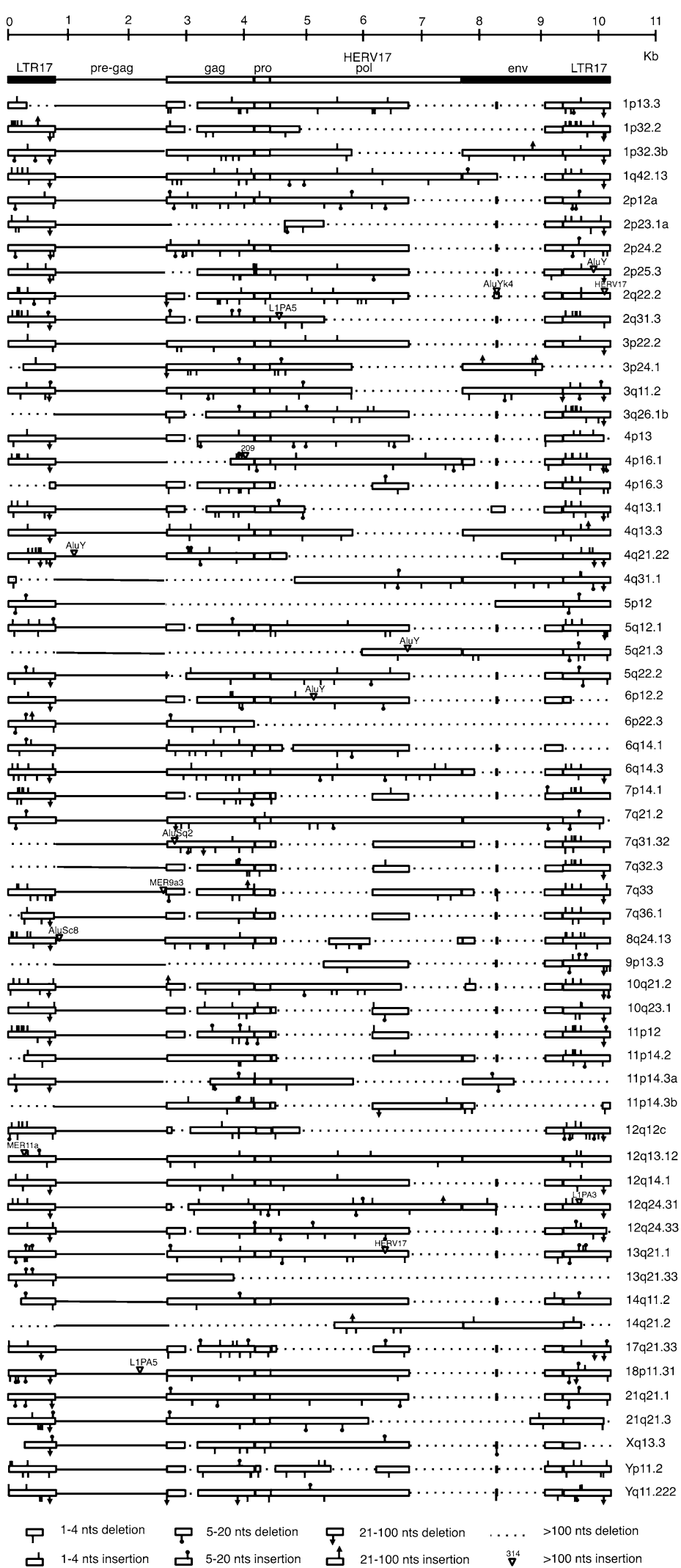

Fig. 2 Insertion and deletions of the 59 proviral sequences $>2.5 \mathrm{~Kb}$ with respect to LTR17-HERV17-LTR17 reference 


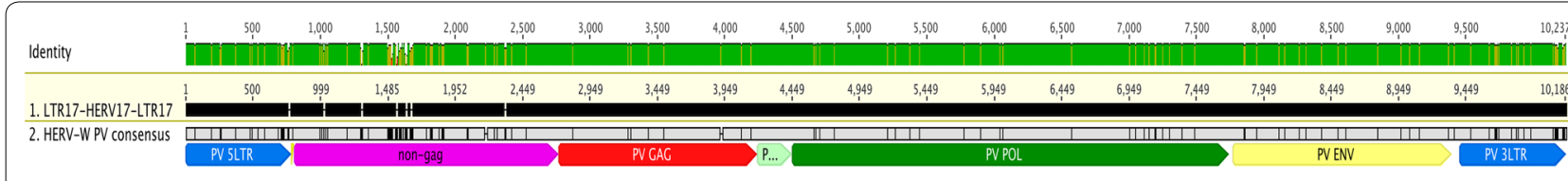

Fig. 3 Comparison between HERV-W RepBase consensus LTR17-HERV17-LTR17 (black) and the proviral dataset generated consensus (grey). Nucleotide identity between the two consensus sequences is represented by the colored upper bar (green $100 \%$ identity; greeny-brown between 100 and $30 \%$ identity; red identity $<30 \%$ ), while single nucleotide differences of the new consensus with respect to LTR17-HERV17-LTR17 are represented with black lines. The retroviral LTRs and genes localization is shown below

subgroup 2 elements showed a lower identity with respect to LTR17 (87\%) and in fact the comparison highlighted the presence of recurrent single nucleotide substitutions. The latter were commonly shared with high frequency in this subset of sequences but not in the other subgroup, and were thus characterized as key mutations for the proposed classification (Table 1). Particularly, in proviral LTRs we identified seven positions with characteristic single nucleotide substitutions with respect to LTR17, which are present in $95-100 \%$ of subgroup 2 members and rarely found (0-3.5 \%) among subgroup 1 members. Moreover, in proviral LTRs tree we observed that subgroup 2 elements seemed divided in two further branches, indicated as type $2 A(n=16)$ and $2 B(n=7)$ (Fig. 2; Table 1). While both shared the recurrent mutation typical of the main subgroup, each one further shows some additional features found in $90-100 \%$ of sequences and rarely present in subgroup 1 elements. These additional mutations were not exclusive of each branch, but were present also in the other subgroup 2 type of elements with frequencies from $19 \%$ up to about $50 \%$ and were thus reported for completeness but not considered for phylogenetic purposes.

The identified key substitutions were then investigated also in the pseudogenic HERV-W dataset, where their strong relation with the sequences distribution in the $\mathrm{NJ}$ trees was confirmed for the first 5 positions (96-100\% frequency in subgroup 2 versus $0-3.5 \%$ in subgroup 1 ), while the last two mutations were shared among about the $75 \%$ of sequences (Table 1). Due to the pseudogenic LTRs truncated structure, the subgroup division was evident in the $3^{\prime}$ LTRs tree (U3-R, positions 1-326 in LTR17) where 5 key positions out of 7 are maintained. The pseudogenic 5' LTRs (R-U5, positions 256-780) harbor instead only the two less represented key positions and showed a more confused topology, underlining the importance of the described substitutions in the phylogenetic asset of the group.

\section{Extended analysis of HERV-W genomic LTRs}

Considering the relevance of LTR structural characteristics for HERV-W classification purposes, we retrieved via Genome Browser BLAT about 800 HERV-W LTRs present in hg19 assembly. This wider dataset has been used to assess the global reliability of the subgroup definition. The NJ tree analysis performed supported our classification, with a tree that resembled the topology observed for proviral and pseudogenic LTRs (Additional file 3: Fig. S2) and showed a comparable distribution of solitary elements between the two subgroups ( $71 \%$ classified as subgroup 1 and $29 \%$ as subgroup 2). When investigated for recurrent substitutions, the key positions defined for subgroup 2 were confirmed as commonly shared in $87-98 \%$ of the subgroup members and rarely present (1-6\%) in the rest of the whole HERV-W LTRs dataset.

\section{Retroviral genes}

The NJ trees built for the retroviral gag, pro, pol and env genes did not highlight the presence of any subgroup (Additional file 2: Fig. S1), and the nucleotide analysis confirmed that the sequences share a comparable grade of homology. This result demonstrated that the phylogenetic relevant variations within the HERV-W group are located in the LTR elements.

A LTR-based classification was previously suggested by Costas, that identified three distinct HERV-W subfamilies named 1,2 and 3, on the basis of nucleotide differences described in a shorter version of the $3^{\prime}$ LTR, with a truncation in correspondence to position 326 of LTR17, typical of pseudogenes [31]. Our data indicate instead that the HERV-W main subgroups are only two: subgroup 1 (associated to Costas subfamily 3 ) and subgroup 2 (related to Costas subfamilies 1 and 2). Subgroup 2 key mutations enclose the 5 mutations observed by Costas plus 2 more in the $3^{\prime}$ LTR terminal portion. With respect to the previous classification, the one we propose is primarily based on a phylogenetic analysis, corroborated by the presence of high frequency key positions found in both $5^{\prime}$ and $3^{\prime}$ full-length LTRs and confirmed for the first time in a comprehensive HERV-W solitary LTRs dataset.

\section{Time of integration}

It is known that, at time of integration, the $5^{\prime}$ - and $3^{\prime}$ LTRs of the same provirus are identical [55] and accumulate random substitution in an independent way. Hence, 


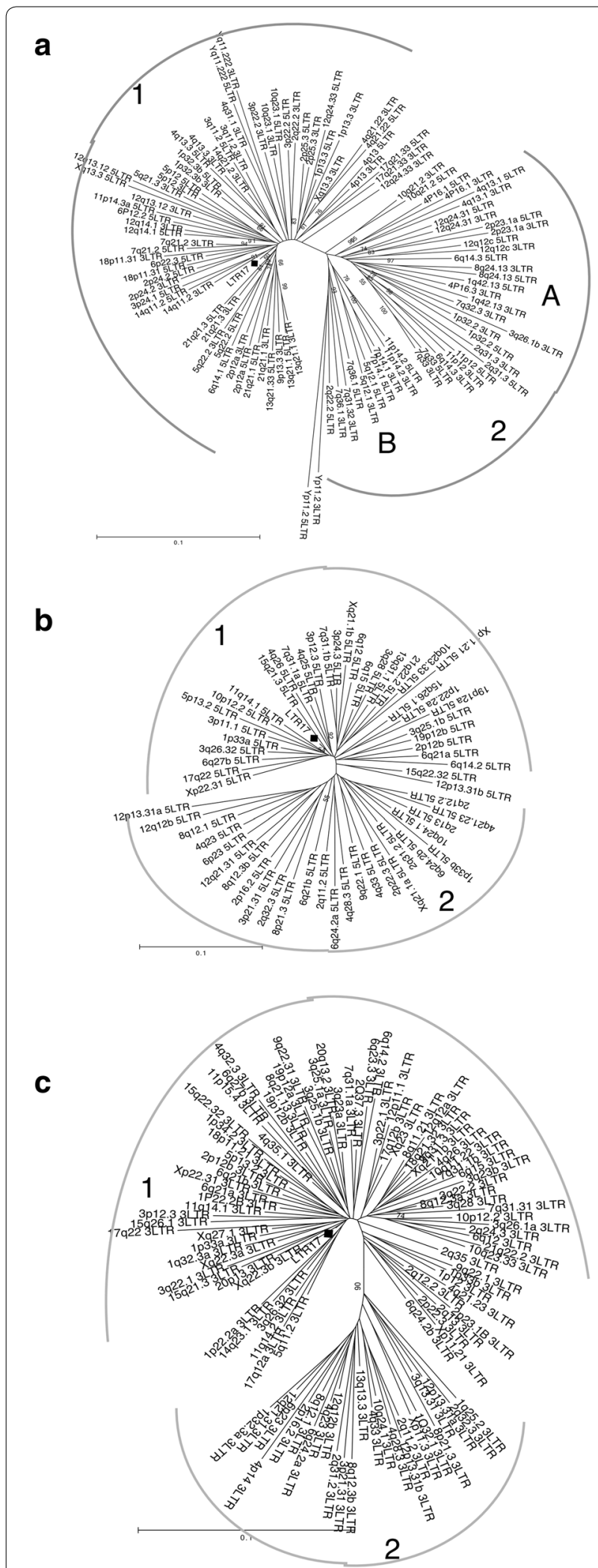

Fig. 4 Neighbor joining trees of HERV-W proviruses $5^{\prime}$ - and $3^{\prime}$ LTRs (a), processed pseudogenes 5' LTRs (b) and processed pseudogenes $3^{\prime}$ LTRs (c). RepBase LTR17 consensus is labeled with a black square to assess the HERV-W group estimated age we assumed for the human genome a substitution rate of $0.13 \% /$ nucleotides/million year [56] and used this rate to assess the action of divergence on each HERV-W sequence. Based on this assumption, we calculated the percentage of divergent nucleotides (D \%) (1) between the $5^{\prime}$ - and $3^{\prime}$ LTRs of each HERV provirus; (2) between each LTR (proviral and pseudogenic) and a generated consensus for each subgroup and (3) between a 150-300 nucleotides region of each HERV-W internal element gag, pro, pol RT, pol IN and env genes (proviral and pseudogenic) and a generated consensus. Regarding the two consensusbased approaches, in consideration that the substitution rate acts randomly on each sequence, the subgroup-generated consensus should ideally represent the ancestral situation.

The obtained divergence values were used to calculate the age of the HERV-W sequences. For all three approaches the calculation is based on the relation $\mathrm{T}=\mathrm{D} \% / 0.13 \%$, where $\mathrm{T}$ is the estimated time of integration (in million years) and $0.13 \%$ is the applied genomic substitution rate per million year. For the divergence between $5^{\prime}$ - and $3^{\prime}$ LTR of the same sequence, the obtained $\mathrm{T}$ value was divided by a factor of 2 , considering that each LTR evolved and accumulated mutations independently. The reported time of integration (Additional file 1: Table S1) has been calculated as the average resulted from the methods used (Fig. 5). In particular, the estimated time of integration of proviral and pseudogenic sequences for both subgroups 1 and 2 (Fig. 5a) describes for the first time the HERV-W dynamic of insertion into the human genome, suggesting that: (1) the first HERV-W integrations involved subgroup 2 and occurred more than 40 million years ago, with a diffusion of proviral and pseudogenic sequences until about 30 million years ago; (2) HERV-W subgroup 1 sequences are significantly younger with respect to subgroup 2 members ( $<<0.0005$ ), and have been acquired mostly between 35 and 25 million years ago, occurring in average about 8 million years later than subgroup 2; (3) it is interesting to note that, for both subgroups, the dissemination of proviruses and processed pseudogenes took place virtually simultaneously. Moreover, despite both subgroups proviruses were processed by the LINE machinery to generate processed pseudogenes, the mechanism was more frequent for subgroup 1 proviruses (1:2.5 ratio with the number of related pseudogenes) than for subgroup 2 integrated elements (1:1 ratio). The reason for this is at the moment unclear. We attempted to connect the single pseudogenic sequences to the original generating proviruses by a phylogenetic analysis of LTRs and major genes, expecting that the pseudogene elements could cluster with their respective HERV-W locus of origin. However, the great majority of pseudogenes clustered 
Table 1 Recurrent mutations in HERV-W subgroup 2 LTRs

\begin{tabular}{llllll}
\hline Position (nt) $^{\mathbf{a}}$ & Substitution $^{\mathbf{b}}$ & Frequency & & \\
\cline { 3 - 6 } & & PV $^{\mathbf{d}}$ subgroup 2 & PG $^{\mathbf{e}}$ subgroup 2 & Solo LTRs subgroup 2 & Subgroup 1 \\
\hline 43 & $\mathrm{C}>\mathrm{T}$ & 100 & 100 & 98 & 0.7 \\
95 & $\mathrm{C}>\mathrm{T}$ & 100 & 95.8 & 96 & 3.4 \\
100 & $\mathrm{~T}>\mathrm{C}$ & 97.3 & 100 & 95 & 2.2 \\
180 & $\mathrm{C}>\mathrm{T}$ & 97.3 & 100 & 95 & 0 \\
254 & $\mathrm{~A}>\mathrm{G}$ & 97.3 & 96 & 87 & 1.4 \\
706 & $\mathrm{~A}>\mathrm{G}$ & 97.4 & 73.3 & 88 & 1.7 \\
765 & $\mathrm{G}>\mathrm{A}$ & 95 & 73.3 & 90 & 1.7
\end{tabular}

\begin{tabular}{|c|c|c|c|c|}
\hline \multirow[b]{2}{*}{ Position (nt) } & \multirow[b]{2}{*}{ Substitution $^{\mathbf{b}}$} & \multicolumn{3}{|l|}{ Frequencyc } \\
\hline & & $\mathrm{PV}^{\mathrm{d}}$ subgroup $2 \mathrm{~A}$ & $\mathrm{PV}^{\mathrm{d}}$ subgroup 2B & Subgroup 1 \\
\hline \multicolumn{5}{|c|}{ Type $2 \mathrm{~A}$ additional mutations } \\
\hline 456 & $C>T$ & 100 & 41.7 & 10.5 \\
\hline 498 & $A>G$ & 92.6 & 33.3 & 3.5 \\
\hline Position (nt) ${ }^{a}$ & Substitution $^{\mathbf{b}}$ & $\mathrm{PV}^{\mathrm{d}}$ subgroup $2 \mathrm{~B}$ & $\mathrm{PV}^{\mathrm{d}}$ subgroup $2 \mathrm{~A}$ & Subgroup 1 \\
\hline \multicolumn{5}{|c|}{ Type $2 B$ additional mutations } \\
\hline 133 & $A>G$ & 100 & 48.1 & 0 \\
\hline 188 & $C>A$ & 90 & 19.2 & 0 \\
\hline 252 & $C>G$ & 90 & 40.7 & 1.7 \\
\hline
\end{tabular}

a Nucleotide positions are referred to RepBase Update LTR17consensus

b Substitutions are indicated with the original nucleotide and the acquired new variant separate by the symbol >

c Relative percentage based on the total of sequences that hold the position in an alignment

d Proviruses

e Pseudogenes

with different proviral loci according to the sequence portion considered (data not shown). Hence, this result, together with the estimated time of diffusion of pseudogenic elements, suggests that the HERV-W processed pseudogenes have acquired a comparable amount of heterogeneity since their mobilization by LINE elements, and it is thus not possible to univocally assign each one to a single proviral locus.

It is important to note that the traditional sole comparison of the two LTRs of the same sequence would not be sufficient for a reliable estimation. In fact: (1) the LTR versus LTR method could not be applied at all to pseudogenic sequences, representing the $63 \%$ of the whole dataset, due to the short region in common between the $5^{\prime}$ - and $3^{\prime}$ LTRs; (2) also in the case of proviral sequences, the lack of one or both LTRs make possible such calculation only for the $70 \%$ of proviral sequences $(23 \%$ of the total HERV-W members). The two additional approaches completed and improved the time of integration estimation, allowing to consider a larger subset of elements (94\% of the total HERV-W members) and to represent also pseudogenes and older and less intact sequences, which were not previously taken into account. Importantly, the combination of multiple divergence calculations provided significant improvements also in age estimation reliability and precision. The expression of each HERV-W sequence time of integration through the use of an averaged value allowed to determine the standard deviation and to reduce estimation biases related to outliers and different selective pressure that are reported to interest LTR elements with respect to the rest of the retroviral genome [38] (Fig. 5b). Data showed that some proviruses had a $0.3-2$ folds higher age estimation when calculated using the LTR versus consensus method as compared with the LTR versus LTR method. Despite the absence of a clear explanation, it is possible to speculate that the exogenous viruses that gave rise to these sequences harbored some nucleotide differences in their LTRs that are not properly represented in the consensus sequence, built on the majority of viruses, leading to an apparent higher amount of mutations. In addition, data showed a higher divergence in the gag, pol-3' (including IN) and $e n v$ portions, leading to a older age estimation with respect to the internal pro and pol-5' regions (Fig. 5c), thus suggesting different mutation rates according to the specific viral portions.

Taken together, these results suggest that the HERV-W group integration started about 40 million years ago at 

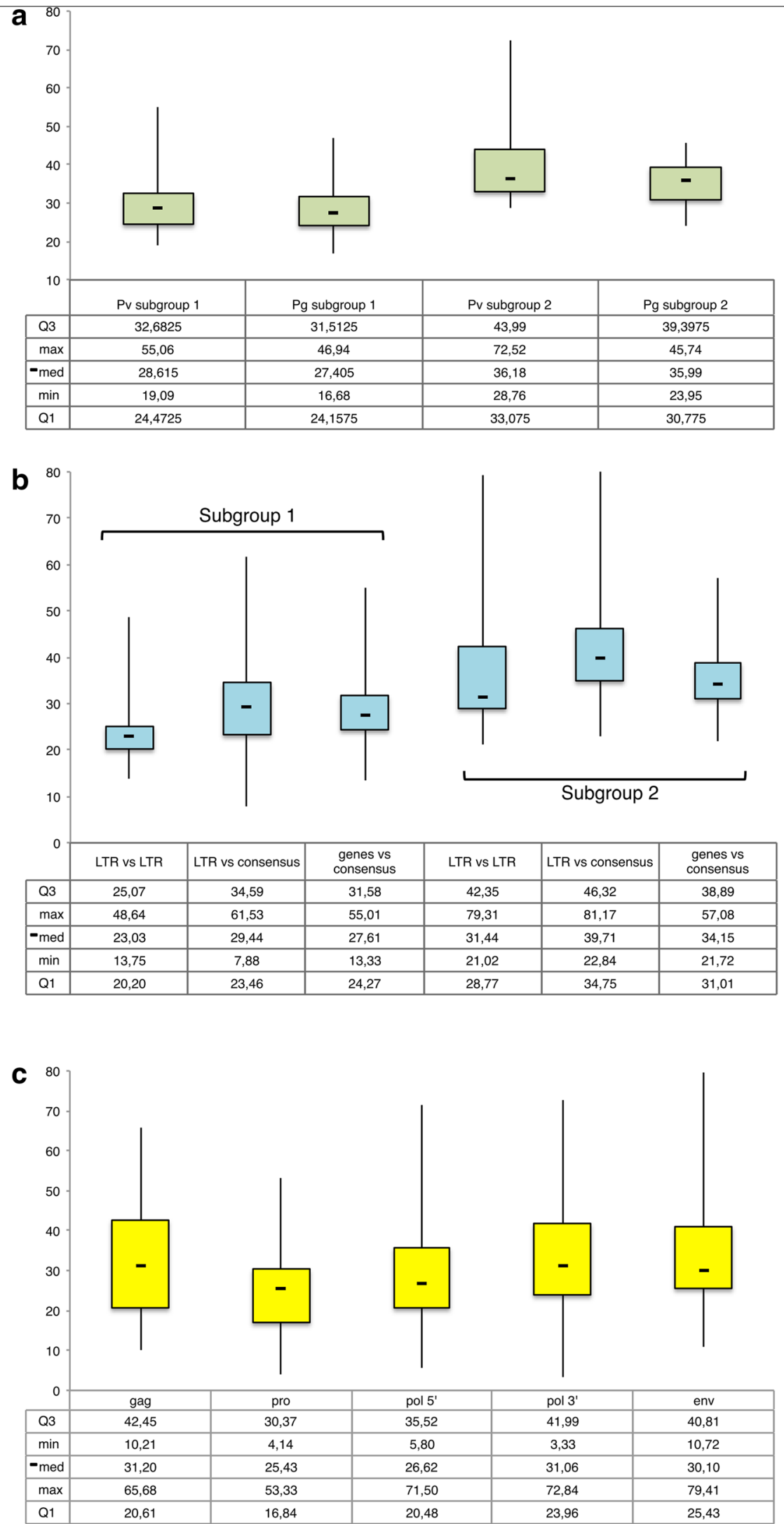
(See figure on previous page.)

Fig. 5 Boxplot representations of HERV-W subgroups divergence based estimated period of integration. The approximated age (in million years) was calculated considering the divergence values between the $5^{\prime}$ - and $3^{\prime}$ LTRs of the same provirus (only for proviral sequences); between each LTR and a generated consensus for each subgroup and between a 150-300 nucleotides region of each HERV-W internal element gag, pro, po/ RT, pol IN and env genes and a generated consensus (proviruses and pseudogenes). a Averaged values of age obtained for each sequence, after the sequences division in proviruses and pseudogenes for each subgroup. b Single method estimations for the two HERV-W subgroups. $\mathbf{c}$ Highlight of the heterogeneous action of the divergence at different genic regions

the time of the Catarrhini primates, after the divergence between New World Monkeys and Old World Monkeys. This is in line with previous studies [31, 57], which were based on the presence of HERV-W PCR products in different Old World Monkey blood samples [57], or on the divergence calculation among HERV-W subfamilies [31], and gave thus just a general overview of primates HERVW group acquisition. In the present study, the time of insertion has been estimated for each single HERV-W locus through at least two different methods of age calculation, providing a precise and exhaustive picture of the group diffusion among primates, with a rather long period of activity that took place until 25-20 million years ago.

The estimated age of the single HERV-W sequences was generally also supported by the identification of each locus orthologous in primates until the Oldest Common Ancestor (O.C.A.) (Additional file 1: Table S1). Results showed that the great majority of sequences are shared from human to Rhesus Macaque (61\%) or to Gibbon (31\%), with an entry that must be occurred after their separation from the Platyrrhini parvorder (40 million years ago) and before their divergence from the evolutionarily younger hominoids, occurred around 30 (Rhesus Macaque) and 20 (Gibbon) million years ago [58]. Few elements were also found starting only since Orangutan (12), Gorilla (3) and Chimpanzee (2) (Additional file 1: Table S1), but in these cases the estimated age was higher than expected. This probably suggests that such sequences were lost in older primates, even though their absence in Rhesus and Gibbon could be also due to a lower efficiency of Genome Browser comparison between the human genome and the most ancient Catarrhini assemblies. Finally, a single HERV-W element was found only in the human genome assembly hg19, on locus $12 \mathrm{q} 13.3$. This data is unexpected because no human specific HERV-W elements have been reported so far, but could not be supported by reliable age estimation due to the shortness of the sequence (about 1500 nucleotides) and the lack of both LTRs.

\section{PBS type and gammaretroviral features}

The PBS type has been historically used to identify the different HERV groups that were commonly designated with the amino acid single letter of the corresponding tRNA. Currently this nomenclature is not considered a sufficient and reliable taxonomic marker, especially because it is not based on HERV phylogeny [5, 59]. In the analyzed HERV-W elements, the PBS was present in 111 sequences and was located approximately 4 nucleotides downstream the $5^{\prime}$ LTR (from nucleotide 4 to 21 in HERV17 consensus). The PBS type of the single sequences is reported in Additional file 1: Table S1, while a graphical overview of the PBS types found in the entire HERV-W dataset and in each subgroup is provided in Fig. 6. In general, Tryptophan (W) was as expected the most common PBS type: it was found in a total of 60 sequences, representing about the $58 \%$ of the identified HERV-W PBSes. Therefore, noteworthy, about half of HERV-W elements analyzed had a non-W PBS type, confirming the relatively low taxonomic value of this feature. Particularly, Arginine PBS was rather common (R, 21), followed by Phenylalanine (F, 9), Isoleucine (I, 4), Serine (S, 3) and Proline (P, 2). Other PBS types found in single HERV-W sequences were Leucine (L), Asparagine $(\mathrm{N})$, Glutamic Acid (E) and Glycine (G). In the remaining eight elements, the PBS sequence was present but it was not possible to unambiguously assign it to a single tRNA. Interestingly, subgroup 1 elements retaining the PBS sequence showed a more homogeneous situation, presenting almost the $100 \%$ of $\mathrm{W}$ or $\mathrm{R}$ as putative tRNA usage. This was expected since the $\mathrm{W}$ codon is the most commonly associated to the HERV-W group, and the $\mathrm{R}$ one differs from it only slightly, and sometimes the two codons may overlap due to a single nucleotide shift in the PBS sequence [5]. Differently, subgroup 2 elements revealed a more heterogeneous $\mathrm{PBS}$ type usage, including all the unusual tRNA sequences and all the ambiguous PBSes with no clear assignment. These atypical PBSes are probably derived from the accumulation of several substitutions, in accordance with the older appearance and the longer permanence of the sequences in primates genome. To summarize the general variation of the PBS sequence within HERV-W group we generated a logo (Fig. 7a) in which the letter height is proportional to the nucleotides conservation at each position. As expected, the TGG starting nucleotides, which are shared by almost all the PBS types, were the most conserved among the 18 bases analyzed. Interestingly the middle portion of the 

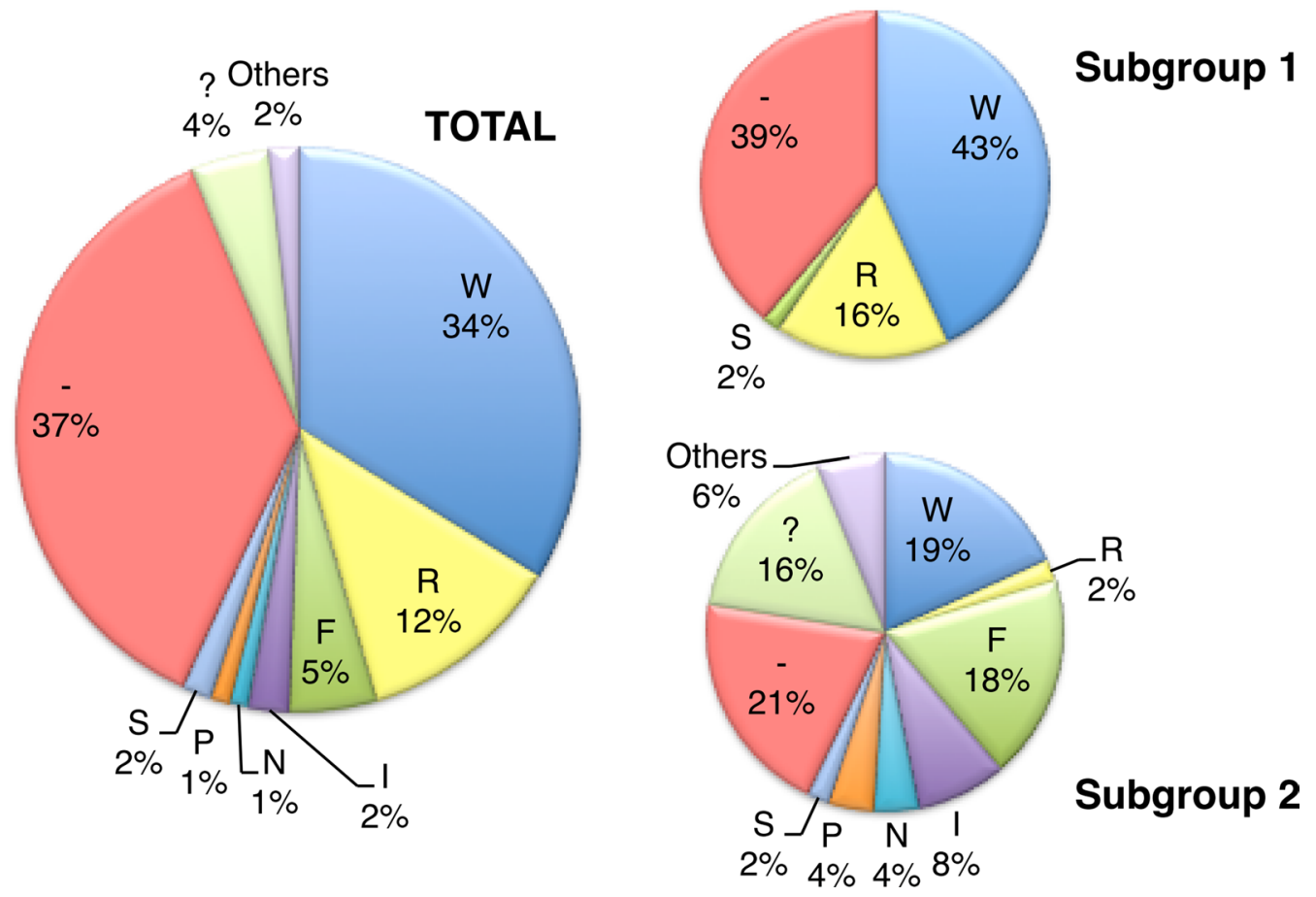

Fig. 6 PBS types among all HERV-W sequences and diversity between subgroup 1 and subgroup 2. The PBS types are identified by the amino acid single letter of the corresponding cellular tRNA. $W=$ tryptophan, $R=$ arginine, $F=$ phenylalanine, $I=$ isoleucine, $S=$ serine, $P=$ proline. "Others" category encloses Leucine (L), Glutamic Acid (E) and Glycine (G), each found only in one sequence. Elements that lost the PBS sequence (-) or with PBS with ambiguous assignation (?) are also included

sequence showed a high variability, especially at positions 4-6 and 11, indicating a rather large diversity of PBSes in HERV-W group.

We have also identified and analyzed structural features typically shared among retroviral sequences within the same genus, that can be used as taxonomic and phylogenetic markers [7]. As previously reported [7], the main gammaretroviral features are (1) one nucleocapsid Zinc finger motif, involved in the retroviral RNA interaction during packaging [60]; (2) the C-terminal polymerase IN GPY/F motif, that binds the host DNA and could have a role in the integration specificity $[61,62]$ and (3) a nucleotide frequency bias determined by the action of encapsidated host RNA editing systems [7].

The gag nucleocapsidic Zinc finger, corresponding to nucleotides 4021-4062 in the RepBase assembled LTR17-HERV17-LTR17 reference sequence, has a typical $\mathrm{CX}_{2} \mathrm{CX}_{4} \mathrm{HX}_{4} \mathrm{C}$ amino acid motif. It was found in almost all sequences that retained the harboring genetic region, with a higher prevalence in proviral sequences that were also the most complete in term of genetic composition. Moreover, noteworthy, a second Zinc finger was identified in $96 \%$ of the sequences (nucleotides 4093-4130). This second Zinc finger has a modified structure with respect to the usual one, showing the loss of one of the variable residues $\left(\mathrm{CX}_{2} \mathrm{CX}_{3} \mathrm{HX}_{4} \mathrm{C}\right)$. The amino acid composition of the two motifs was highly conserved as shown in Fig. 7b. The presence of a second Zinc finger was not previously reported for HERV-W group, and its structure is consistent with the second Zinc finger found in a subset of HERV-H sequences, another gammaretroviral HERV group [63]. However, while for HERV-H a correlation between the presence of this second motif and the age of sequences was proposed, for HERV-W we could not observe such correlation (data not shown).

The IN domain contains a GPY/F motif, a stretch of conserved amino acids with the general WXnGPYXV structure corresponding to nucleotides 7501-7521 in the reference sequence. Considering that the $\mathrm{C}$-terminal part of the polymerase gene was deleted in $85 \%$ of sequences, in the remaining few members the GPY/F feature was found with a $100 \%$ frequency. Also for this feature the logo analysis showed a conserved amino acid sequence (Fig. 7c).

Regarding the nucleotide composition, HERV-W members present a weak bias in purines, tending to be richer in Adenine (about $30 \%$ ) and poorer in Guanine (around $22 \%$ ) (data not shown). Among Gammaretroviruses an impoverishment of $\mathrm{G}$ nucleotide was previously observed for HERV-H group in association with an higher content 


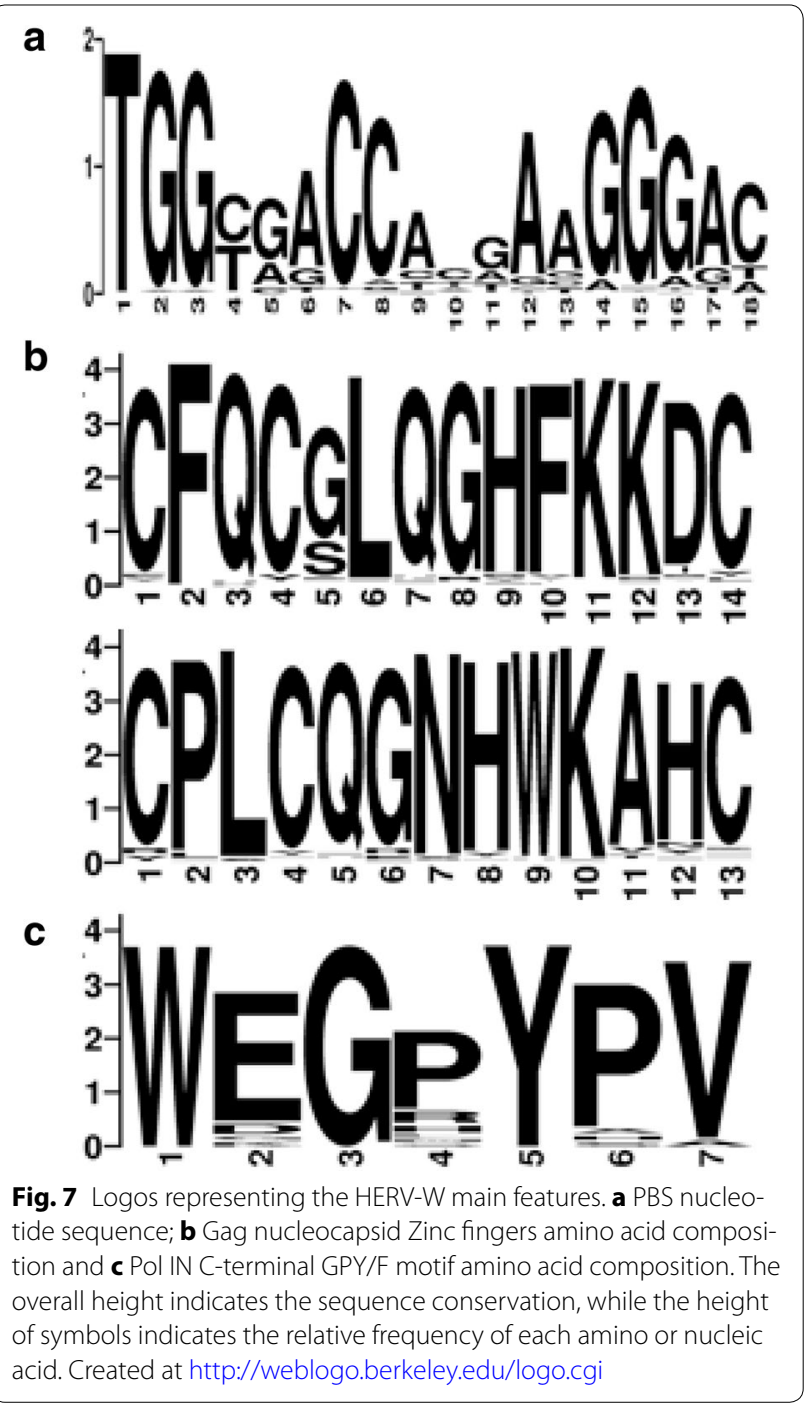

of Cytosine [63], while the G to A hypermutation condition was reported for HERV-K group [64] and is a well known effect of the APOBEC3 defensive action against HIV-1 Lentivirus [65]. Hence, it is possible to speculate that this editing system could have played a role as a control mechanism to limit HERV-W and other endogenous elements mobility during evolution [66], also considering APOBEC3 ability to greatly inhibit the LINE mediated transposition of other retroelements [67].

\section{Genomic context of insertion}

The current major field of HERVs investigation is their expression and coding capacity, however, the impact of these sequences on the host depends also on their genetic surrounding. The context of integration can, in fact, modulate HERVs physiology, and HERV sequences inserted in proximity of human genes are known to be able to influence their expression [39-41, 43, 45, 48, 49]. As reported for other HERV groups [68], the analysis of the genomic context of all 213 HERV-W confirmed that the majority of sequences are located in intergenic regions, with the exception of 80 elements inserted into human genes.

In particular, 55 elements $(26 \%)$ are inserted into human coding genes, mostly exclusively into intronic regions (53/55) (Table 2). These elements showed a strong anti-sense orientation with respect to the surrounding gene (43/55) that was more frequent for pseudogenic sequences than for proviruses (84 and $67 \%$, respectively). The fact that HERV-W intronic elements are present mostly in antisense orientation could reflect a bias due to an evolutionary and post-insertional selection [69]. Noteworthy, while most of the identified sequences were already characterized for their genomic context [20, 70], 8 of them are reported for the first time to be inserted in human genes. Based on Genome Browser annotations, pseudogene $21 \mathrm{q} 22.2$ resulted from an overlap in antisense orientation with the first two exons of IGSF5 gene, that produces a protein involved in junction and adhesion formation, and with a corresponding IGSF5 highly similar mRNA found in placental tissues (AK092516). Interestingly, more than half of the 55 genes that held HERV-W sequences were reported to be positively associated with a disease or a pathologic trait, that in most cases affect the neurological and the cardiovascular systems (Table 2). Considering that the genomic context of integration and the orientation of HERV-W sequences appeared to influence their expression [70], the present mapping of those elements may aid in the understanding the potential effects of these integrations on human health and to direct further investigations to the genes involved.

In addition, 25 HERV-W loci were integrated into 29 human non-coding genes (Table 3) of which the great majority (22) are reported here for the first time. These elements were mostly inserted into regions associated with the production of long non-coding RNA (lincRNA) and microRNA (miRNA), regulatory molecules that operate on different levels of gene expression. These HERV-W proviruses (6), pseudogenes (17) and undefined sequences (2) showed different characteristics with respect to the loci observed into coding regions. Firstly, despite even in this case a majority of intron localization (26) was observed, 8 of them were co-localized with 9 exons, frequently situated at the transcriptional start site of the non-coding gene. Secondly, in this case the antisense bias was not present, since 19 out of the 29 noncoding genes showed the same orientation with respect to the HERV-W inserted elements. These observations suggest that the LTRs of these HERV-W could provide 
Table 2 HERV-W genomic context: insertions into human coding genes

\begin{tabular}{|c|c|c|}
\hline HERV-W & Human gene & Gene or relative protein function and associations \\
\hline $1 \mathrm{p} 34.2(+)$ & HIVEP3 Int $1(-)$ & Transcription factor, binds Ig and T-cell receptors recombination signal \\
\hline $1 \mathrm{q} 25.2(-)$ & $\underline{\text { RASAL2 } \operatorname{lnt} 1(+)^{b}}$ & RAS superfamily of small GTPases protein activator like. Associations: BMI, weight \\
\hline $1 q 42.13^{*}(-)$ & $\underline{Z N F 678} \operatorname{lnt} 2(+)^{\mathrm{a}, \mathrm{b}}$ & Zinc Finger protein. Associations: body height \\
\hline $2 \mathrm{p} 23.1 \mathrm{a}^{*}(+)$ & LCLAT1 Int $2(+)^{\mathrm{b}}$ & Predominantly remodels anionic phospholipids in endoplasmic reticulum \\
\hline $2 \mathrm{p} 16.2(+)$ & ASB3 Int $1 / 2(-)^{b}$ & Suppressor of cytokine signaling proteins and their binding partners \\
\hline $2 \mathrm{q} 22.2^{*}(-)$ & $\underline{\text { KYNU }} \operatorname{lnt} 2(+)^{\mathrm{a}, \mathrm{b}}$ & NAD cofactors biosynthesis from tryptophan. Associations: body height, cholesterol, schizophrenia \\
\hline $2 \mathrm{q} 24.3(-)$ & $\underline{\operatorname{COBLL1} 1} \operatorname{lnt} 2(-)^{\mathrm{a}}$ & $\begin{array}{l}\text { Cordon bleu WH2 repeat protein-like 1. Associations: BMI, Cholesterol, HDL, triglycerides, stroke, response } \\
\text { to statin therapy, anthropometric sexual dimorphism }\end{array}$ \\
\hline $2 \mathrm{q} 31.2 \mathrm{a}(-)$ & $\boldsymbol{A G P S} \operatorname{Int} 1(+)^{b}$ & [603051] Mutations are cause of rhizomelic chondrodysplasia punctata type 3 \\
\hline $2 q 35(-)$ & $\underline{D I R C 3} \operatorname{lnt} 1(-)^{\mathrm{b}}$ & Disrupted in renal carcinoma long non-coding RNA. Associations: diabetes mellitus \\
\hline $3 p 22.2^{*}(-)$ & SLC22a14 Int $1(+)^{b}$ & Solute carrier transmembrane protein \\
\hline $3 q 22.1(-)$ & 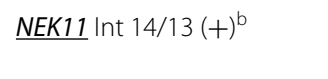 & $\begin{array}{l}\text { Never In mitosis kinase. Involved in DNA replication and } \mathrm{G}_{2} / \mathrm{M} \text { checkpoint response to DNA damage. } \\
\text { Related to embryonic lethality and preeclampsia }\end{array}$ \\
\hline $3 q 23 b(+)$ & XRN1 Int $1(-)^{\mathrm{b}}$ & Exoribonuclease involved in Long noncoding RNA decapping and miRNA regulation \\
\hline $3 q 26.32(+)$ & ZMAT3 Int 2/3 (-) $)^{a, b}$ & Zinc finger matrin. Acts as a bona fide target gene of p53/TP53 \\
\hline $4 \mathrm{p} 16.3^{*}(-)$ & ZNF595 Int $3(+)$ & Zinc finger protein. Function as transcription factor \\
\hline $4 p 16.1^{*}(+)$ & ACOX3 Int $1(-)^{a, b}$ & Oxidizes the CoA-esters of 2-methyl-branched fatty acids \\
\hline $4 \mathrm{q} 31.3(+)$ & $\boldsymbol{A R F I P 1} \operatorname{lnt} 2(+)^{\mathrm{b}}$ & ADP ribosylation factor interacting protein1. [605928] Enhance the cholera toxin activity \\
\hline $5 q 12.1^{*}(+)$ & $\underline{D E P D C 1 B} \operatorname{lnt} 2(-)^{\mathrm{b}}$ & Significantly upregulated in nonsmall cell lung carcinoma cell lines (reduced patient survival) \\
\hline $5 q 22.2^{*}(+)$ & ACOT13 Int $1(+)$ & Acyl-CoA thioesterase. Involved in regulation of lipid composition and metabolism \\
\hline $6 q 12(+)$ & $\boldsymbol{E Y S} \operatorname{lnt} 13(-)^{\mathrm{b}}$ & [612424] In photoreceptor layer: mutated in autosomal recessive retinitis pigmentosa \\
\hline $6 q 14.3 a^{*}(-)$ & $\underline{T B X 18} \operatorname{lnt} 7(-)^{\mathrm{a}, \mathrm{b}}$ & Role in embryonic development. Associations: cholesterol, coronary disease \\
\hline $6 \mathrm{q} 21 \mathrm{a}(+)$ & $\underline{\text { ATG5 }} \operatorname{lnt} 6(-)^{\mathrm{a}, \mathrm{b}}$ & Autophagy related apoptosis specific protein. Associations: lipoproteins, LES \\
\hline $6 q 21 b^{\circ}(+)$ & PDSS2 Int $2(-)^{b}$ & $\begin{array}{l}\text { Prenyl (decaprenyl) diphosphate synthase, subunit 2. Synthesizes the side-chain of coenzyme Q. [610564] } \\
\text { Coenzyme Q10 deficiency, primary, 3: fatal encephalomyopathy and nephrotic syndrome }\end{array}$ \\
\hline $6 q 21 c(+)$ & $\underline{S L C 16 A 10} \operatorname{Int} 1(+)^{a, b}$ & $\begin{array}{l}\mathrm{Na}^{(+)} \text {-independent transport of aromatic amino acids across the plasma membrane. Associations: choles- } \\
\text { terol, LDL }\end{array}$ \\
\hline $6 \mathrm{q} 24.2 \mathrm{a}(-)$ & $\underline{A I G 1} \operatorname{lnt} 1(+)^{\mathrm{b}}$ & Androgen-induced. Associations: C-reactive protein, insulin, myocardial infarction \\
\hline $7 \mathrm{p} 21.1(-)$ & $B Z W 2 \operatorname{lnt} 3(+)$ & Homo sapiens basic leucine zipper and W2 domains 2 \\
\hline $7 p 14.1^{*}(-)$ & SUGCT/C7orf10 Int $1(+)^{b}$ & $\begin{array}{l}\text { [609187] Mutations are associated with glutaric aciduria type III. Others: BMI, fat distribution, cardiomegaly, } \\
\text { coronary disease, pancreatic and prostatic neoplasms }\end{array}$ \\
\hline $7 q 31.1 a(+)$ & $\underline{N R C A M} \operatorname{lnt} 2(-)^{\mathrm{a}}$ & Neuronal Cell Adhesion Molecule. Associations: autism, obsessive compulsive disorder, schizophrenia \\
\hline $7 q 31.1 b(-)$ & $\boldsymbol{F O X P 2} \operatorname{lnt} 2(+)^{a, b}$ & $\begin{array}{l}\text { [605317] Required for development of speech and language regions of the brain during embryogenesis. } \\
\text { Associated to speech-language disorders }\end{array}$ \\
\hline $8 p 21.3(+)$ & $\underline{S L C 18 A 1} \operatorname{Int} 10 / 11(-)^{b}$ & $\begin{array}{l}\text { Involved in vesicular transport of biogenic amines. Associations: bipolar disorder, major depressive disor- } \\
\text { der }\end{array}$ \\
\hline $8 q 12.3 a(-)$ & NKAIN3 Int $3(+)$ & $\begin{array}{l}\mathrm{Na}+/ \mathrm{K}+\text { transporting ATPase interacting proteins. Associations: mental competency, neuroblastoma, } \\
\text { stroke }\end{array}$ \\
\hline $8 q 12.3 b(+)$ & CYP7B 1 Int $1(-)^{b}$ & $\begin{array}{l}\text { [603711] Cyp450 enzyme. Associations: bile acid synthesis congenital defect, spastic paraplegia. Others: } \\
\text { Alzheimer disease, lipoproteins, schizophrenia }\end{array}$ \\
\hline $8 q 21.11(+)$ & UBE2 $\boldsymbol{W} \operatorname{lnt} 2(-)^{\mathrm{b}}$ & $\begin{array}{l}\text { Ubiquitin-conjugating enzyme. Along with ubiquitin-activating (E1) and ligating (E3) enzymes, coordi- } \\
\text { nates the ubiquitin addition to proteins. [614277] Interacts with FANCL and regulates the monoubiquit- } \\
\text { ination of Fanconi anemia protein FANCD2 }\end{array}$ \\
\hline $8 q 21.13(+)$ & ZNF704 Int $2(-)^{b}$ & Zinc finger protein \\
\hline 9p24.1 (+) & PTPRD Int $12(-)^{b}$ & $\begin{array}{l}\text { Protein tyrosine phosphatase, receptor type, D. [601598] Restless Legs Syndrome. Associations: asthma, } \\
\text { BMI, cholesterol, lipids, lipoproteins, triglycerides, diabetes }\end{array}$ \\
\hline $9 p 13.3^{*}(-)$ & $\underline{C D 72} \operatorname{lnt} 1(-)^{\mathrm{a}, \mathrm{b}}$ & B-cell proliferation and differentiation antigen. Associations: lupus erythematosus \\
\hline $10 q 23.33(-)$ & CYP2C19 Int $6(+)^{b}$ & $\begin{array}{l}\text { [124020] Cyp450 enzyme, responsible for therapeutic agents metabolism. Associated to metabolic defects } \\
\text { and variants }\end{array}$ \\
\hline 10q24.1 (-) & ENTPD1 Int $1(+)^{\mathrm{b}}$ & [601752] Triphosphate Diphosphohydrolase. Associated with Spastic Paraplegia \\
\hline $11 p 14.2^{*}(-)$ & ANO3 Int $14(+)^{a, b}$ & $\begin{array}{l}\text { [610110] May act as a chloride channel. Associations: Dystonia 24. Others: bmi, obesity, c-reactive protein, } \\
\text { cholesterol, coronary disease, schizophrenia }\end{array}$ \\
\hline $11 q 14.1(-)$ & $\operatorname{AAMDC} \operatorname{lnt} 2(+)^{\mathrm{b}}$ & Adipogenesis associated Mth938 domain containing \\
\hline
\end{tabular}


Table 2 continued

\begin{tabular}{|c|c|c|}
\hline HERV-W & Human gene & Gene or relative protein function and associations \\
\hline $11 q 14.2(-)$ & PRSS23 Int $2(+)^{b}$ & Encodes a conserved member of the trypsin family of serine proteases \\
\hline $12 \mathrm{p} 13.31 \mathrm{~b}(-)$ & RIMKLB Int $5(+)^{\mathrm{b}}$ & Catalyses ATP-dependent condensation of NAA and glutamate to produce NAAG \\
\hline $12 q 23.3(+)$ & $S L C 41 A 2 \operatorname{lnt} 1(-)$ & Solute carrier family 41 member 2 \\
\hline $13 q 13.3(+)$ & $A L G 5 \operatorname{lnt} 7 / 8(-)^{b}$ & Participates in N-linked glycosylation of proteins \\
\hline $14 q 11.2^{*}(+)$ & TCRA Int $1(+)^{\mathrm{b}}$ & T cell receptor alpha locus \\
\hline $14 q 21.2^{*}(-)$ & FAM179B Int $7(+)$ & Homo sapiens family with sequence similarity 179 member B \\
\hline $14 q 23.1(+)$ & C14orf37 Int $4(-)^{b}$ & Associations: attention deficit disorder with hyperactivity \\
\hline $17 q 12 a(+)$ & SLFN14 Int $3(-)^{\mathrm{b}}$ & Implicated in regulation of cell growth and T-cell development (studies in mouse \\
\hline $17 q 12 b^{\circ}(-)$ & $\underline{A C A C A} \operatorname{lnt} 2 / 6(-)^{\mathrm{b}}$ & Biogenesis of long-chain fatty acid. Associations: BMl, breast cancer \\
\hline $17 q 22(-)$ & $\underline{\operatorname{STXBP4}} \operatorname{lnt} 8(+)^{\mathrm{b}}$ & $\begin{array}{l}\text { Translocation of transport vesicles from cytoplasm to plasma membrane, like the insulin-stimulated GLUT4 } \\
\text { translocation in adipocytes. Associations: BMI, cholesterol }\end{array}$ \\
\hline 19p12a (+) & ZNF90 Int $1(+)^{\mathrm{b}}$ & Zinc finger protein 90. May be involved in transcriptional regulation. [603973] \\
\hline $19 \mathrm{q} 13.2 \mathrm{a}(+)$ & ZNF780A Ex $9(-)^{\mathrm{b}}$ & Zinc finger protein $780 \mathrm{~A}$ \\
\hline $19 q 13.2 b(-)$ & CYP2A7 in $1(-)^{\mathrm{b}}$ & Cytochrome P450, family 2, subfamily A, polypeptide 7 \\
\hline $21 q 22.2(-)$ & $\underline{\text { IGSF5 }}$ Ex $1-2, \operatorname{lnt} 1(+)^{\mathrm{b}}$ & $\begin{array}{l}\text { Participates at tight-junctions (kidney, gut) or acts as adhesion molecule (testis). Associations: coronary } \\
\text { disease, lipoproteins, Parkinson disease, stroke }\end{array}$ \\
\hline Xp11.21 (-) & FAAH2 Int $7(+)^{b}$ & Degradation and inactivation of bioactive fatty acid amides \\
\hline Yq11.222* (+) & CD24 Int $1(-)$ & Mature granulocytes and B cells surface antigen \\
\hline
\end{tabular}

Proviruses and undefined sequences are labeled respectively with * and ${ }^{\circ}$. For HERV-W sequences and genes, the strand direction is reported into round brackets. Bold genes are listed as OMIM diseases associated and the relative accession number is reported into square brackets. Underlined genes are reported to be positive associated with specific phenotypes in UCSC Gene annotations

a Already reported in Li et al. [70]

b Already reported in Schmitt et al. [20]

regulatory signals for lincRNA, as already highlighted for HERV LTRs in general [71]. The great majority of noncoding genes are still uncharacterized, but some elements were reported to be associated with post-transcriptional regulation (MIR5684) or related to proteins involved in lipid transfer and proteolytic activity (STARD7-AS1 and PRSS23). Overall, the percentage of HERV-W sequences inserted into human genes (36\%) is higher than the percentage of bases spanned by human genes $(24 \%)[1,72]$ suggesting that integration events could have been biased for genic or against intergenic regions.

Finally, the HERV-W elements were evaluated for the possibility to bind cellular transcription factors (TFs) that normally interact with the host DNA. The analysis was based on the data obtained through ENCODE Transcription Factors ChIP-seq and Factorbook databases, and the TFs with higher score (ranging from 800 to 1000) were considered to be the most reliable (Table 4). Results showed that 16 HERV-W elements could tentatively bind cellular TFs, including POLR2A, MAFK, E2F1 and TCF7L2. Twelve of these elements were proviruses, and 7 of them plus 1 pseudogene were inserted into human coding genes. The higher representation of proviral sequences is probably due to the presence of complete LTR structures. Despite the fact that the detection of predicted TF binding sites is not enough to suggest a possible transcription, their presence in HERV-W elements that are co-localized with human genes could potentially have an effect on the transcription of such genes and need to be further investigated at expression level.

\section{Env putative proteins analysis}

Due to its physiological role, the ERVWE1/Syncytin-1 ORF has been characterized in detail in terms of structure and functional domains, as recently reviewed [73]. Hence we wanted to compare the Syncytin-1 precursor ORF and its features with respect to the most complete env genes found in our dataset, in order to predict the conservation of those sites reported to be involved in Syncytin-1 protein in vivo functions. Within our HERV-W elements, in addition to the Syncytin-1 locus ORF (7q21.2, 538 aa), we found 16 full-length or near full-length (longer than $1.4 \mathrm{~Kb}$ ) env genes, 3 more than previously reported with similar parameters [74], and 10 conserved but shorter env genes (from 1398 to 801 nucleotides). These env genes manual translation led to the correspondent putative proteins (puteins), with a length range of 483-559 aa and 267-466 aa, respectively (Table 5). These Env puteins were obtained from different reading frames in the often-damaged env gene candidates, and are thus just a bioinformatics model useful to evaluate the predicted domains structure. Env puteins were aligned and analyzed with respect to the Syncytin-1 amino acid sequence (NCBI reference NP_055405.3), 
Table 3 HERV-W genomic context: insertions into human non-coding genes

\begin{tabular}{|c|c|c|}
\hline HERV-W & Human gene & Gene function and associations \\
\hline $1 \mathrm{p} 12(-)$ & LOC101929147Int $4(+)$ & Uncharacterized antisense long non-coding RNA \\
\hline $1 \mathrm{p} 13.3^{*}(-)$ & TCONS_00000271 Int $3(+)$ & Large intergenic non coding RNA \\
\hline $1 \mathrm{q} 32.1(-)$ & LOC284581 Int $1(+)^{\mathrm{b}}$ & Uncharacterized antisense long non-coding RNA \\
\hline $2 q 11.2(-)$ & STARD7-AS1 Int1 $(+)^{\mathrm{b}}$ & StAR-related lipid transfer domain protein 7 antisense long non coding RNA (LOC285033) \\
\hline $2 q 24.3(-)$ & TCONS_00004484 Int $1(-)$ & Long intergenic non coding RNA \\
\hline $2 \mathrm{q} 31.2 \mathrm{~b}(+)$ & MIR548 N Int $1(+)^{\mathrm{b}}$ & Homo sapiens microRNA 548n \\
\hline $3 q 25.1 b(+)$ & CLRN1-AS1 Int $1(+)$ & CLRN1 antisense non-coding RNA \\
\hline $4 \mathrm{p} 13^{*}(-)$ & TCONS_00007753 Int $1(-)$ & Long intergenic non coding RNA \\
\hline $4 q 23(-)$ & LOC100507053 Int $1(+)$ & Uncharacterized antisense long non-coding RNA \\
\hline $4 q 28.3(+)$ & TCONS_00007833 Int 1 (-) & Long intergenic non coding RNA \\
\hline $4 q 32.3(+)$ & MIR5684 Int $2(+)$ & MicroRNA involved in post-transcriptional regulation of gene expression \\
\hline $6 q 15(-)$ & TCONS_00011526 Ex 1, Int $1(-)$ & Long intergenic non coding RNA \\
\hline $6 \mathrm{q} 27 \mathrm{a}^{\circ}(+)$ & $\begin{array}{l}\text { TCONS_12_00024517 Int 2, Ex } 3(+) \\
\text { TCONS_12_00024518 Int 1, Ex } 2(+) \\
\text { TCONS_12_00024519 Int } 1(+)\end{array}$ & Long intergenic non coding RNAs \\
\hline $7 p 14.2^{*}(+)$ & DQ594967 Ex 1(- $)^{b}$ & Antisense non coding RNA \\
\hline $8 q 12.1(-)$ & $\begin{array}{l}\text { TCONS_00015019 Int } 1(-) \\
\text { AC022555.1 (-) }\end{array}$ & $\begin{array}{l}\text { Long intergenic non coding RNA } \\
\text { Pseudogene }\end{array}$ \\
\hline $9 \mathrm{p} 21.3(+)$ & LOC441389 Int 5 Ex $6(+)^{b}$ & Uncharacterized long non-coding RNA \\
\hline $10 q 11.22(-)$ & TCONS_00017977Int $1(-)$ & Long intergenic non coding RNA \\
\hline $11 q 14.2(-)$ & PRSS23 Int $2(+)$ & Protease serine 23 near-coding RNA \\
\hline $11 \mathrm{q} 23.3(-)$ & TMPRSS4-AS1 Int $2(-)^{\mathrm{b}}$ & Antisense non-coding RNA \\
\hline $13 q 21.33^{*}(+)$ & LINC00383 Ex 1, Int $1(+)$ & Long intergenic non coding RNA \\
\hline $13 \mathrm{q} 31.3^{\circ}(+)$ & TCONS_00021873 Int $2(+)$ & Long intergenic non coding RNA \\
\hline $21 \mathrm{q} 21.1^{*}(-)$ & MIR548XHG Ex 1, Int $1(-)$ & MIRNA548X host gene long non-coding RNA \\
\hline $14 q 22.1(+)$ & AL163953.3 Int $3(+)$ & Long non-coding RNA \\
\hline 19p12d (+) & AK125686 Int $2(-)^{b}$ & Antisense non coding RNA \\
\hline $\mathrm{Xq13.3*}(-)$ & $\begin{array}{l}\text { TCONS_00016997 Ex 1-2, Int } 1(+) \\
\text { AL451105.1 (-) }\end{array}$ & $\begin{array}{l}\text { Long intergenic non coding RNA } \\
\text { Pseudogene }\end{array}$ \\
\hline
\end{tabular}

Proviruses and undefined sequences are labeled respectively with * and ${ }^{\circ}$. For HERV-W sequences and genes, the strand direction is reported into round brackets

a Already reported in Li et al. [70]

${ }^{b}$ Already reported in Schmitt et al. [20]

showing a general accumulation of nucleotide substitutions, insertion and deletion. This led to the frequent occurrence of multiple premature internal stop codons as well as many frameshifts, which prevents the effective production of a complete protein (Additional file 4: Fig. S3, Additional file 5: Fig. S4).

Noteworthy, seven Env puteins conserved a coding sequence without internal stop codons. Among them, three env genes (4q13.3, 5q11.2 and Xp22.31) are theoretically long enough to encode a complete protein (Additional file 4: Fig. S3). However, even if uninterrupted, those ORFs showed changes of reading frame with respect to the Syncytin-1 translation mode. 20q13.2 (483 aa) and 4q21.22 (320 aa) sequences are the most conserved with respect to Syncytin-1, presenting no stop codons and only one frameshift between positions 441-442 and 75-76, respectively. Xq22.3b (542 aa) and 9q22.31 (267 aa) present indeed no frameshifts but showed a single internal stop codon (position 39 and 149, respectively) that could potentially be reverted with a single point mutations, as already demonstrated ex vivo for Xq22.3b N-trenv [75].

Regarding the amino acid composition, all investigated Env puteins accumulated several substitutions, leading to a general average identity of about $85 \%$ with respect to Syncytin-1 sequence. To evaluate the puteins possible biological activity, we have characterized in detail the motifs known to be mostly involved in the Syncytin-1 physiological function. Primarily, the envelope precursor must be processed into the mature SU and TM units, with a proteolytic cleavage that occurs at the Furin Cleavage Site conserved RKNR motif. The mutation of this conserved domain has been reported to abrogate the proteolytic cleavage and the fusogenic activity of Env proteins, that exhibited also delayed 
Table 4 HERV-W genomic context: transcription factor (TF) binding sites

\begin{tabular}{|c|c|c|c|}
\hline HERV-W & TF recognized & Position & Score $(0-1000)$ \\
\hline $2 p 12 a^{*}$ & POLR2A & chr2:76098843-76099352 & 803 \\
\hline $2 q 22.2^{*}$ & E2F1 & $\begin{array}{l}\text { chr2:143661226- } \\
143661546\end{array}$ & 958 \\
\hline $3 p 22.2^{*}$ & CTCF & chr3:38331061-38331485 & 900 \\
\hline \multirow[t]{2}{*}{$4 p 16.1 *$} & POLR2A & chr4:8429472-8430544 & 1000 \\
\hline & TCF7L2 & chr4:8424096-8424592 & 922 \\
\hline \multirow[t]{3}{*}{$6 \mathrm{p} 12.2^{*}$} & TFAP2C & chr6:52783052-52783485 & 1000 \\
\hline & FOXA2 & chr6:52783244-52783462 & 848 \\
\hline & STAT3 & chr6:52784270-52784579 & 808 \\
\hline \multirow[t]{2}{*}{$6 q 14.3^{*}$} & STAT3 & chr6:85427859-85428174 & 1000 \\
\hline & CEBPB & chr6:85427862-85428118 & 817 \\
\hline 7q21.1* & TCF7L2 & chr7:92103429-92103733 & 1000 \\
\hline \multirow[t]{2}{*}{ 7q31.1a } & TCF7L2 & $\begin{array}{l}\text { chr7:107981247- } \\
\text { 107981830 }\end{array}$ & 1000 \\
\hline & E2F1 & $\begin{array}{l}\text { Chr7:107981308- } \\
\text { 107981897 }\end{array}$ & 1000 \\
\hline $7 q 33 *$ & YY1 & $\begin{array}{l}\text { chr7: } 34270591- \\
134271127\end{array}$ & 1000 \\
\hline $7 q 36.1^{*}$ & FOXA1 & $\begin{array}{l}\text { chr7:149370177- } \\
\text { 149370408 }\end{array}$ & 1000 \\
\hline $9 q 22.1$ & TCF7L2 & chr9:91556701-91556965 & 1000 \\
\hline \multirow[t]{2}{*}{$10 \mathrm{q} 21.2^{*}$} & GATA3 & $\begin{array}{l}\text { Chr10:62797340- } \\
62797529\end{array}$ & 1000 \\
\hline & E2F1 & $\begin{array}{l}\text { chr10:62796837- } \\
62797697\end{array}$ & 806 \\
\hline \multirow[t]{4}{*}{$10 \mathrm{q} 23.1^{*}$} & GATA1 & $\begin{array}{l}\text { Chr10:86284672- } \\
86285183\end{array}$ & 1000 \\
\hline & MAFK & $\begin{array}{l}\text { Chr10:86285572- } \\
86285911\end{array}$ & 1000 \\
\hline & MAFF & $\begin{array}{l}\text { Chr10:86285647- } \\
86285793\end{array}$ & 1000 \\
\hline & TBL1XR1 & $\begin{array}{l}\text { Chr10:86284785- } \\
86285185\end{array}$ & 824 \\
\hline \multirow[t]{2}{*}{$10 q 24.1$} & TAL1 & chr2:97480654-97480806 & 928 \\
\hline & TEAD4 & $\begin{array}{l}\text { chr10:97480630- } \\
\quad 97480810\end{array}$ & 859 \\
\hline $10 q 21.3$ & MAFK & $\begin{array}{l}\text { chr10:65805045- } \\
65805364\end{array}$ & 1000 \\
\hline \multirow[t]{2}{*}{ 21q21.1* } & TFAP2C & $\begin{array}{l}\text { chr21:20128637- } \\
20128925\end{array}$ & 1000 \\
\hline & YY1 & $\begin{array}{l}\text { chr21:20131977- } \\
\text { 20132464 }\end{array}$ & 859 \\
\hline
\end{tabular}

Data obtained from Genome Browser Encode Transcription Factor ChIP-seq database

Proviruses and undefined sequences are labeled respectively with * and ${ }^{\circ}$. Bold loci are the one inserted into human genes

kinetics of appearance on the membrane compared to the wild-type envelope [76]. The RKNR motif of the HERV-W puteins was frequently mutated at the first position, mostly with the conversion of $\mathrm{R}$ residue to $\mathrm{C}$ or $\mathrm{H}$ ( $73 \%$ of analyzed ORFs), but was maintained in 7 sequences. After cleavage,
SU and TM mature proteins are then linked through a covalent disulphide bond between the SU CWIC and the TM CX6CC motifs. While the TM domain showed a high degree of amino acid homology with respect to Syncytin-1, in the SU motif we found an I > M substitution in $100 \%$ of sequences. Another fundamental step that drives the fusion activity is the interaction between the SU N-terminal 124 aa receptor binding domain and a human sodium-dependent neutral amino acid transporter (hASTC1 or hASTC2), which acts as type D mammalian retrovirus receptor. In the binding domain, the SDGGGX2DX2R motif was recognized to be essential for the receptor contact, and was found in the $58 \%$ of the sequences. The Syncytin-1 fusogenic activity is held by the TM portion, that includes a fusion peptide and a fusion core formed by the amino- and carboxy-terminal heptad repeats. In Env puteins the fusion peptide sequence was characterized by at least one substitution, with residue 332 (A) that was mutated in all sequences into an R or a $\mathrm{G}$ (and in one case into an $\mathrm{E}$ ). Also the fusion core was affected by several mutations localized in both heptad regions, like the residue $433 \mathrm{R}>\mathrm{Q}$ substitution that is present in 25 out of 26 carboxy-terminal repeats. Interestingly, the 75 amino acids long heptad repeat region showed also a higher concentration of internal stop codons, harboring $50 \%$ of the total stop codons found in the analyzed puteins. Moreover, in traditional Env proteins the fusogenic activity is prevented by an inhibitory $\mathrm{R}$ peptide that is located in the TM intracytoplasmic tail and is normally removed by viral proteases. In Syncytin-1 a four amino acid deletion at the LQMV cleavage site made the protein constitutively competent for fusion [77]. This mutation was not present in any other analyzed HERV-W Env putein. Finally, the Syncytin-1 TM subunit also contains a conserved immunosuppressive domain that was thought to possibly contribute towards maternal immunotolerance [12] even though following findings suggested the absence of this activity [14]. In any case, in the selected Env puteins this domain presents several amino acid substitutions and in 5 sequences a premature termination at position 383 . Hence, with respect to locus 7q21.2 Syncytin-1 protein, the other HERV-W loci Env puteins resulted highly defective, especially in sites involved in known physiological functions. However, despite these mutations, they may still be able to produce shorter proteins with a biological significance and/or a role in disease development, as observed for other HERV sequences [78].

Due to its maintenance despite the presence of huge recurrent flanking deletions affecting the $85 \%$ of HERV$\mathrm{W}$ env genes, also the small env portion of about 30 nucleotides at position 8289-8318 was translated and compared with respect to Syncytin-1. As shown in Additional file 6: Fig. S5, all the 138 HERV-W elements that maintained this portion showed recurrent amino 
Table 5 Env puteins analysis

\begin{tabular}{|c|c|c|c|}
\hline Sequence & $\begin{array}{l}\text { ORF length (amino } \\
\text { acids) }\end{array}$ & Stop & Shift \\
\hline $7 q 21.2^{*}$ & 538 & 0 & 0 \\
\hline $1 \mathrm{p} 32.3 \mathrm{~b}^{*}$ & 559 & 3 & 2 \\
\hline $6 q 21 a$ & 552 & 4 & 2 \\
\hline $15 q 21.3$ & 543 & 2 & 2 \\
\hline $4 q 13.3^{*}$ & 542 & 0 & 2 \\
\hline$\underline{5 q 11.2}$ & 542 & 0 & 4 \\
\hline $5 q 21.3^{*}$ & 542 & 6 & 2 \\
\hline $12 q 13.12^{*}$ & 542 & 2 & 1 \\
\hline $14 q 21.2^{*}$ & 542 & 3 & 1 \\
\hline Xq22.3b & 542 & 1 & 0 \\
\hline $3 q 11.2^{*}$ & 541 & 1 & 3 \\
\hline $4 q 31.1^{*}$ & 541 & 2 & 2 \\
\hline $17 q 12 b$ & 540 & 2 & 2 \\
\hline Xp22.31 & 529 & 0 & 6 \\
\hline $20 q 13.2$ & 483 & 0 & 1 \\
\hline 11 p15.4 & 475 & 2 & 3 \\
\hline $3 p 24.1^{*}$ & 466 & 2 & 0 \\
\hline 9q31.3 & 462 & 3 & 1 \\
\hline $3 q 23 a$ & 453 & 2 & 1 \\
\hline 4932.3 & 443 & 1 & 2 \\
\hline $1 \mathrm{q} 32.3 \mathrm{a}$ & 361 & 0 & 3 \\
\hline $5 p 12^{*}$ & 355 & 6 & 0 \\
\hline 1p34.2 & 352 & 2 & 1 \\
\hline Xq27.1 & 352 & 4 & 1 \\
\hline$\underline{4 q 21.22^{*}}$ & 320 & 0 & 1 \\
\hline$\underline{17 q 12 a}$ & 296 & 0 & 2 \\
\hline $9 q 22.31$ & 267 & 1 & 0 \\
\hline
\end{tabular}

Proviruses are labeled with *, Syncytin-1 ORF is highlighted in bold. Underlined sequences retain an ORF without internal stop codons; italic sequences did not present frameshifts

acid substitutions. In particular, the $\mathrm{N}$ in position 3 was changed in 136/138 sequences, substituted by $\mathrm{H}$ in $93 \%$ of the elements; while the $\mathrm{V}$ in position 8 was substituted in 135 sequences, showing a I in $90 \%$ of cases. This prevalence indicates that Syncytin-1 protein probably represents the exception, suggesting an unreported functional relevance of this short domain.

\section{MSRV sequences homology with HERV-W elements}

To complete the overview on the HERV-W presence and impact on human genome it was useful to consider also the proposed association with MS disease. In fact, the first HERV-W member was originally identified as cDNA sequences derived from particle-associated RNA in MS patients cultured cells $[79,80]$. Those sequences were subsequently indicated as MS associated Retrovirus (MSRV) [11, 81, 82], proposed to be an exogenous competent member of the HERV-W group, related to the MS development [83-86]. Other reports, however, remarked the uncertain nature of MSRV $[28,87,88]$ and proposed that some of these CDNA sequences could arise from the recombination of different HERV-W loci transcripts [89]. According to this hypothesis, such recombination could happen through RT switching templates, likely during in vitro PCR amplification, that is a common complication during the analyses of transcribed elements [90]. In particular, Laufer et al. proposed that six sequences, previously published as MSRV elements, could be traceable to a single HERV-W locus or a recombination between two HERV-W loci transcripts [89]. Since other four sequences published as MSRV (accession numbers: AF009668, AF009666, AF009667 and AF123880) [81, 82] were not analyzed for possible HERV-W origin, having a more complete HERV-W database we analyzed them as described [89], including the six previously investigated MRSV sequences as internal control (Table 6). The analysis confirmed that AF127227, AF127228, AF123882, and AF123881 have high identity with a single HERV-W locus, while AF127229 and AF331500 could origin from recombination of two HERV-W loci transcripts. Similarly, AF009666 pro-pol and AF009667 pol mRNAs showed high identity with the HERV-W locus 1p34.2 (99.5\% similarity) and 17q22 (98.2 \% similarity), respectively, while AF123880 5' LTRpre gag mRNA showed sequence identity with three HERV-W loci (5p12, 3p24.1 and 3q26.32) with a similarity for each component ranging from 98 to $100 \%$. Finally, AF009668 pro-pol mRNA showed a more complex identity pattern with a high degree of mosaicism that seems to involve several HERV-W loci: 1p34.2, 2p12a, 2p24.2, 6q27b, 6q15 and 3p12.3. Interestingly, AF009668 shares a $95 \%$ similarity with AF135487, a retroviral-related sequence identified to be schizophrenia associated and also mapped to multiple sites [91]. Moreover, we performed the same analysis with the four MSRV DNA probes used to characterize the HERV-W placental expression. These probes were obtained through RT-PCR from RNA particles found in synoviocyte culture supernatants and pellets of a Rheumatoid arthritis patient (AF072494 pol- and AF072498 env-probes respectively) and in B lymphocyte culture and plasma of a MS patient (AF072496 gag- and AF072497 pro-probes respectively) [11]. Both AF072494 pol- and AF072496 gag-probes showed high identity with HERV-W 6q21b locus $(99.6 \%$ similarity), while AF072497 pro- and AF072498 envprobes were highly identical to locus 1p34.2 (99.2\%) and Xq22.3b (99.5\%), respectively.

The MSRV sequences containing an env gene (or a portion of it) and showing highest identity with one of the 
Table 6 HERV-W loci homology of previously described MSRV sequences and probes

\begin{tabular}{|c|c|c|c|c|}
\hline MSRV GenBank entry & HERV-W locus/loci & Query cover & $\begin{array}{l}n^{\circ} \text { of discordant } \\
\text { bases }\end{array}$ & Mapped portion in LTR17-HERV17-LTR17 \\
\hline AF127227 (544 bp) & $3 q 23 a^{*}(99.5 \%)$ & $1-544$ & 3 & env (8208-8752) \\
\hline AF127228 (1932 bp) & $X q 22.3 b^{*}(99.6 \%)$ & $1-1932$ & 9 & pol-env (5444-5838 and 7682-9200) \\
\hline \multirow[t]{2}{*}{ AF127229 (2004 bp) } & $3 p 12.3^{*}(99.9 \%)$ & $1-1084$ & 2 & \multirow{2}{*}{$\begin{array}{l}\text { pol-env-3' LTR (5452-6792 and 8290-8318 and } \\
\text { 9115-9732) }\end{array}$} \\
\hline & $18 q 21.32 *(99.9 \%)$ & $1055-2004$ & 2 & \\
\hline AF123882 (2477 bp) & $12 q 21.3 *(99.8 \%)$ & $1-2477$ & 7 & pol-env (5720-8199) \\
\hline \multirow[t]{2}{*}{ AF331500 (1629 bp) } & $X q 22.3 b^{*}(99.7 \%)$ & $1-1332$ & 4 & env (7720-9348) \\
\hline & $5 p 12^{*}(99.4 \%)$ & $1308-1629$ & 2 & \\
\hline AF123881 (1511 bp) & $3 q 26.32^{*}(99.9 \%)$ & $1-1511$ & 2 & gag-pro (2765-4269) \\
\hline \multirow[t]{6}{*}{ AF009668 (2304 bp) } & 1 p34.2 (99.1 \%) & $1-633$ & 6 & pro-pol (4178-6480) \\
\hline & $2 \mathrm{p} 12 \mathrm{a}(100 \%)$ & $623-736$ & 0 & \\
\hline & $2 \mathrm{p} 24.2(100 \%)$ & $717-871$ & 0 & \\
\hline & $6 q 27 b(98.5 \%)$ & $837-1424$ & 11 & \\
\hline & $6 q 15(97.2 \%)$ & $1415-1763$ & 10 & \\
\hline & 3p12.3 (99.4\%) & $1719-2304$ & 4 & \\
\hline AF009666 (324 bp) & 1 p34.2 (99.5 \%) & $1-324$ & 3 & pro-pol (4178-4521) \\
\hline AF009667 (118 bp) & $17 q 22(98.2 \%)$ & $1-118$ & 2 & pol (5031-5148) \\
\hline \multirow[t]{3}{*}{ AF123880 (1003 bp) } & $5 p 12(99.6 \%)$ & $1-203$ & 1 & $5^{\prime} \operatorname{LTR}(255-803)$ \\
\hline & $3 p 24.1(100 \%)$ & $198-593$ & 1 & \\
\hline & $3 q 26.32(98 \%)$ & $592-1003$ & 11 & \\
\hline AF072494 pol probe (678 bp) & $6 q 21 b(99.6 \%)$ & $1-678$ & 5 & pol (4660-5338) \\
\hline AF072496 gag probe (536 bp) & $6 q 21 b(99.6 \%)$ & $1-536$ & 2 & pre gag-gag(2706-3199) \\
\hline AF072497 pro probe (364 bp) & 1 p34.2 (99.2 \%) & $1-364$ & 4 & pro-pol (4166-4522 and 5641-5549) \\
\hline AF072498 env probe (591 bp) & Xq22.3b (99.5\%) & $1-591$ & 3 & env (8606-9196) \\
\hline
\end{tabular}

Previously published MSRV sequences and probes (column 1) were analyzed for their homology to one/more HERV-W locus/loci by BLAT search, considering the best match in human genome (reported in column 2 near to each HERV-W element). The MSRV elements portion similar to HERV-W locus/loci (column 3) as the number of discordant nucleotides with respect to the identified HERV-W locus/loci (column 4) and the correspondent positions in the LTR17-HERV17-LTR17 reference (column 5) were obtained through Mafft alignment and Geneious platform analysis. MSRV sequences were characterized through the analysis of each element with respect to the whole HERV-W dataset with Recco software

* Already investigated by Laufer et al. [89]

- $95 \%$ similarity with AF135487, a retroviral-related sequence reported to be schizophrenia associated and mapped to multiple sites

HERV-W loci analyzed for Env puteins were manually translated and aligned with the correspondent HERV-W Env putein and the Syncytin-1 protein for further comparison (Additional file 7: Fig. S6). Interestingly, with respect to the Syncytin-1 sequence the HERV-W puteins and the correspondent MSRV putein shared the great majority of amino acid substitutions, and often the same amino acid change was common to all sequences analyzed. AF127227 and 3q23a share the same frameshift at position 270 of Syncytin-1 sequence. Moreover, AF127227 and AF127228 showed an internal stop codon at the same position observed in 3q23a and Xq22.3b, respectively (position 39, W in Syncytin-1). Differently, AF331500 lacks this internal stop codon presenting, like Syncytin-1, a W in this position. As already observed for HERV-W, also MSRV Env puteins showed at least one amino acid change in all domains relevant to Syncytin-1 biological activity. Given the proposed MSRV Env proteins role in pathogenesis, the presence of shared recurrent substitutions, possibly preventing the MSRV Env puteins functionality as compared to Syncytin-1, opens further questions that will have to be addressed. Overall, while more MSRV RNA expression studies are needed, the here reported HERV-W genomic map and characterization is a further step to properly assess the MSRV/HERV-W role in the context of MS.

\section{Conclusions}

Since the discovery of Syncytin-1 role in placentation [11-13, 92], a great attention has been dedicated to the expression potential of the HERV-W group, trying to further understand their impact on the host. Many studies were focused on HERV-W correlations with several human diseases, primarily represented by MS [15-21, 28,75 , and reviewed in 76] and other major neurological pathologies such as schizophrenia and bipolar disorder $[23,25,93]$. Despite this broad investigation, no certain 
correlations between HERV-W group expression and any human disease has been confirmed. Also in the major field of MS the findings are still highly discordant [28]. One of the problems faced in this scenario is still the unfortunate lack of a complete and updated description of the HERV-W sequences in the human genome, their genomic background and a detailed knowledge of HERV-W single members. Such information could help in better interpreting the wide range of collected HERV-W expression data.

Therefore, using more updated genome data and a double bioinformatics identification approach, we performed an analysis on the GRCh37/hg19 assembly identifying a total of 213 HERV-W unambiguously classified members. Each HERV-W sequence has been precisely localized and characterized in term of structure, phylogeny and evolution, allowing to specifically identify the uniqueness of each HERW-W single member, and highlighting various non-previously reported characteristics of the group.

Firstly, we observed several nucleotide differences of HERV-W members with respect to the assembled LTR17HERV17-LTR17 reference that was built on a small number of sequences and therefore does not properly represent the entire group. Secondly, we classified the HERV-W members into two subgroups through a LTRs phylogenetic analysis strongly supported by the identification of key mutated positions in both LTRs, shared by the majority (from $95 \%$ up to $100 \%$ ) of sequences within the same subgroup. Beside LTRs mutations relevant for classification purposes, the subgroups comparisons showed single nucleotides differences along the whole retroviral sequence. For this reason we propose here two new consensuses, one for each subgroup (Additional file 8: File S1), that in our opinion better represent the overall HERV-W group composition.

In the present study, for the first time, the period of insertion has been estimated for each HERV-W locus through at least two different methods of age calculation. This provided a precise and exhaustive picture of the group diffusion among primates, and brought important improvements in the method reliability and applicability. Moreover, the analysis showed significantly different dynamics in the two subgroups diffusion, pointed out also by the analysis of the PBS type variability.

The analysis of structural features described for Gammaretroviruses [7] in HERV-W single members allows to characterize them for the first time in term of prevalence and sequence conservation among the group. Noteworthy, in addition to the traditional Zinc finger motif [94], we found a previously unreported second putative Zinc finger with an unusual structure, lacking one variable residue. Another interesting feature reported here for the first time is the presence of a weak bias in the HERV$\mathrm{W}$ elements purine amount, with enrichment in $\mathrm{A}$ and a consequent underrepresentation of $\mathrm{G}$.
With regards to the group genomic context, we provide an updated overview of $80 \mathrm{HERV}-\mathrm{W}$ elements inserted into human genes and the predicted capacity to bind cellular TFs. In particular, 55 HERV-Ws were found into coding genes, 8 more than what previously observed [20, $70]$, while 25 elements were inserted in human non-coding genes, of which the great majority (22) are reported here for the first time.

Env putein analysis led us to identify and functionally characterize 16 full-length or near full-length env genes, 3 more than previously reported [74], and 10 conserved but shorter env genes. Although the relative puteins resulted highly defective and mutated in comparison to Syncytin-1, these genes may still be able to produce shorter proteins with a biological significance, as observed for other HERV sequences [78].

In the light of the debated connection between HERVW loci expression and MS disease, we investigated the elements known as MSRV in order to evaluate their identity with respect to one or more HERV-W loci in agreement to what has been previously reported [89]. Our results confirmed that the majority of MSRV related sequences have from 97 to $100 \%$ identity with one single HERV-W locus, but more complex pattern of identity, apparently involving 3 or even 6 loci, were also observed. Furthermore, the comparison between MSRV Env puteins and the highest identical HERV-W loci puteins showed common amino acid substitutions with respect to Syncytin-1, that affect all domains reported as relevant for its biological activity.

In conclusion, this report provides, to our knowledge, the most exhaustive and updated overview to date on HERV-W group in terms of structure, evolution and context of integration into the human genome, revealing that this polymorphic multicopy family is not only represented by the single HERV-W member Syncytin-1. We showed that HERV-W elements were acquired by primates during a rather long period, and evolved within and with their genome that exerted a selective pressure leading to the modification of HERV-W structures, including the previously shown co-option of one member for an important physiological function [12, 13]. Overall, the here presented characterization of the HERV-W composition and their genomic context of insertion, will be essential to investigate the effects that, beside protein expression, HERV-W can exert in different tissues both in physiological conditions as well as putative involvement in human disease development and clinical manifestations and to better define their real impact and contribution to our genome.

\section{Methods}

\section{HERV-W identification and localization}

The 213 HERV-W sequences were collected from GRCh37/

hg19 assembly using a double approach that binds (1) 
the hg19 assembly analysis by the ReTe program package [50] and (2) a traditional BLAT search [51] in the UCSC Genome Browser database [95] using the RepBase Update [52] assembled LTR17-HERV17-LTR17 consensus as a query. The elements found by both approaches have then been confirmed as HERV-W based on (1) Repeat Masker analysis of the HERV-W sequence and its genomic flanking portions, (2) structural alignment and comparison with respect to the HERV-W group RepBase reference LTR17HERV17-LTR17 and (3) phylogenetic trees; in order to avoid misclassifications or incomplete sequences inclusion.

HERV-W solitary LTRs were retrieved by UCSC Genome Browser BLAT search using LTR17 as a query, and kindly provided by Professor Jens Mayer (Saarland University).

\section{Sequences alignment and structural characterization}

The HERV-W nucleotide composition was characterized in detail with respect to the RepBase Update assembled LTR17-HERV17-LTR17 reference by multiple alignments performed with Mafft on line program, version 7 [96] and the subsequent analysis on Geneious bioinformatics software platform, version 8.1.4 [97]. All insertion and deletions were annotated, and the presence of other repetitive elements was reported.

\section{Phylogenetic trees}

Phylogenetic trees were built with Mega Software, version 6 [98] using pairwise deletion and p-distance method with 500 bootstrap replications. In addition to HERV-W nucleotidic sequences and RepBase Update LTR17 and HERV17 consensus, each tree initially included a HERV9 generated consensus [5]. This was initially made in order to identify and eliminate eventual members of this HERV-W related family.

\section{Time of integration estimation}

The age of the single HERV-W members was estimated based on the percentage of divergent nucleotides (D \%) between (1) 5' - and $3^{\prime}$ LTRs of each provirus, (2) proviral and pseudogenic single LTRs and a generated consensus for each subgroup, and (3) proviral and pseudogenic 150-300 nucleotides gag, pro, pol RT, pol IN and env portions and a generated consensus for each subgroup. The divergence values were estimated on Mega 6 through Kimura 2-parameter corrected pairwise distances excluding gaps and $\mathrm{CpG}$ dinucleotides. The $\mathrm{D} \%$ have then been used according to previous methodologies [56] to estimate the time of integration $(\mathrm{T})$ assuming an human genome substitution rate of $0.13 \% /$ nucleotides/million years, with the formula $\mathrm{T}=\mathrm{D} / 0.13$. For the proviral $5^{\prime}$ - versus $3^{\prime}$ LTR divergence a factor of 2 was applied assuming that each LTR evolved independently into the genome $(T=D / 0.13 / 2)$. The final age of each sequence was expressed as average of the estimated time of integration obtained, excluding those value with a standard deviation $>20 \%$.

\section{PBS and gammaretroviral features representation}

The presence and composition of the PBS nucleotide sequence and of the nucleocapsidic Zinc finger and C-terminal polymerase IN GPY/F amino acid motifs were analyzed using Mafft alignment and Geneious platform. The grade of conservation at each position was represented with a logo built from WebLogo at http://weblogo.berkeley.edu [99]. The PBS assignation to the correspondent human tRNA type was made by similarity analysis with respect to a tRNA library built from the Transfer RNA database (tRNAdb) of Leipzig University [100] and from the PBS library provided by Professor Jonas Blomberg [5].

\section{Genomic context}

The genomic context of each HERV-W sequence was characterized by the integration of their genomic coordinates with the UCSC Genome Browser Genes and Genes prediction tracks [101-103]. The elements colocalized with human genes were further analyzed by BLAST search after the activation of OMIM, UCSC, RefSeq and Gencode genes annotations [104]. The presence of TFs binding sites were characterized by the integration of HERV-W members genomic coordinates with the UCSC Genome Browser Regulation Encode Txn Factor ChIp tracks $[105,106]$. TFs binding sites were considered reliable in the presence of a score ranging from 800 to 1000 .

\section{Env puteins analysis}

The env selected genes were translated in all possible frames using Geneious platform. The alignment with respect to ERVWE1/Syncytin-1 precursor (NCBI reference sequence NP_055405.3) was performed on Mafft and allowed to reconstruct the complete protein and to annotate all frameshifts and stop codons. The structural and functional relevant domains were analyzed on Geneious platform.

\section{Analysis of MSRV sequences}

Previously published MSRV sequences and probes were retrieved from GeneBank and analyzed by BLAT search for the best matching HERV-W locus/loci based on nucleotide sequence similarity in GRCh37/hg19 assembly. Alignments of MSRV sequences and the relative best matching HERV-W elements were manually inspected on Geneious platform, and discordant positions were annotated. The HERV-W locus/loci homology was then confirmed through the software Recco [107] with respect to our whole HERV-W dataset as described [89]. 


\section{HERV-W consensus sequences generation}

The HERV-W group and subgroups consensus sequences were generated from our HERV-W dataset using Geneious bioinformatics software platform, version 8.1.4 [97].

\section{Additional files}

Additional file 1: Table S1. HERV-W elements identification in Human Genome assembly GRch37/hg19.

Additional file 2: Fig. S1. Phylogenetic neighbor joining trees of proviral and pseudogenic (A) gag, (B) pol and (C) env genes. RepBase HERV17 consensus is labeled with a black square.

Additional file 3: Fig. S2. Phylogenetic neighbor joining trees of about 800 HERV-W LTRs retrieved from GRCh37/hg19 assembly. LTR17 consensus is labeled with a black square.

Additional file 4: Fig. S3. Env puteins analysis with respect to Syncytin-1 (locus 7q21.2). In Syncytin-1 ORF the domains mostly involved in the protein structure and function are annotated: Leader Peptide $(L P)$, Binding Domain motif (BD), SU and TM disulphide bounds motifs (dS), Furin cleavage site (FCS), fusion core N- and C- terminal Heptad Repeats (NHR and (HR), Immunosuppressive domain (IM), Transmembrane unit (TM), Intracytoplasmic Tail (CYT) and the relative Syncytin-1 specific deletion (LQMV del). In Env puteins amino acid substitutions and internal stop codons with respect to Syncytin-1 are labeled with colored and black lines, respectively. The reading frames are reported below each sequence with a number and an arrow.

Additional file 5: Fig. S4. Residues-visible version of Env puteins analysis with respect to Syncytin-1 (locus 7q21.2). In Syncytin-1 ORF the domains mostly involved in the protein structure and function are annotated: Leader Peptide (LP), Binding Domain motif (BD), SU and TM disulphide bounds motifs (dS), Furin cleavage site (FCS), fusion core $\mathrm{N}$ - and C-terminal Heptad Repeats (NHR and CHR), Immunosuppressive domain (IM), Transmembrane unit (TM), Intracytoplasmic Tail (CYT) and the relative Syncytin-1 specific deletion (LQMV del). In Env puteins amino acid substitutions and internal stop codons with respect to Syncytin-1 are labeled with colored and black squares, respectively. The reading frames are reported below each sequence with a number and an arrow. The orange arrow indicates the Env portion frequently maintained in presence of flanking huge recurrent deletions.

Additional file 6: Fig. S5. HERV-W Env puteins maintained portion comparison with respect to Syncytin-1 ammino acid sequence. Amino acid substitutions are labeled with colored and squares. A consensus sequence is shown above Syncytin-1.

Additional file 7: Fig. S6. MSRV Env puteins analysis with respect to the most similar HERV-W loci Env puteins and Syncytin-1 (locus 7q21.2). In Syncytin-1 ORF the domains mostly involved in the protein structure and function are annotated: Leader Peptide (LP), Binding Domain motif (BD), SU and TM disulphide bounds motifs (dS). Furin cleavage site (FCS), fusion core N- and C-terminal Heptad Repeats (NHR and CHR), Immunosuppressive domain (IM), Transmembrane unit (TM), Intracytoplasmic Tail (CYT) and the relative Syncytin-1 specific deletion (LQMV del). In Env puteins amino acid substitutions and internal stop codons with respect to Syncytin-1 are labeled with colored and black squares, respectively. The reading frames are reported below each sequence with a number and an arrow. The orange arrow indicates the Env portion frequently maintained in presence of flanking huge recurrent deletions.

Additional file 8: File S1. HERV-W proposed consensus in Fasta format. HERV-W group general proviral consensus sequence (HERVW_PVcons) and HERV-W subgroups 1 and 2 consensus sequences (HERVW_SG1 Cons and HERVW_SG2cons, respectively) generated starting from our HERV-W sequences dataset.

\section{Authors' contributions}

NG performed the analysis and wrote the manuscript. MC participated in the analysis and in the writing. JB and ET conceived and coordinated the study. All authors helped edit the manuscript. All authors read and approved the final manuscript.

\section{Author details}

${ }^{1}$ Department of Life and Environmental Sciences, University of Cagliari, Cittadella Universitaria di Monserrato SS554, 09042 Monserrato, Cagliari, Italy.

${ }^{2}$ Department of Medical Sciences, Uppsala University, Uppsala, Sweden.

${ }^{3}$ Istituto di Ricerca Genetica e Biomedica, Consiglio Nazionale delle Ricerche (CNR), Monserrato, Cagliari, Italy.

\section{Acknowledgements}

Authors thank Jens Mayer for useful discussions and critical observations.

\section{Competing interests}

The authors declare that they have no competing interests.

Received: 18 April 2016 Accepted: 23 August 2016

Published online: 09 September 2016

\section{References}

1. Lander ES, Linton LM, Birren B, Nusbaum C, Zody MC, Baldwin J, Devon K, Dewar K, Doyle M, FitzHugh W, Funke R, Gage D, Harris K, Heaford A, Howland J, Kann L, Lehoczky J, LeVine R, McEwan P, McKernan K, Meldrim J, Mesirov JP, Miranda C, Morris W, Naylor J, Raymond C, Rosetti M, Santos R, Sheridan A, Sougnez C, et al. Initial sequencing and analysis of the human genome. Nature. 2001;409:860-921.

2. International Human Genome Sequencing Consortium. Finishing the euchromatic sequence of the human genome. Nature. 2004;431:931-45.

3. Bannert N, Kurth R. The evolutionary dynamics of human endogenous retroviral families. Annu Rev Genom Hum Genet. 2006;7:149-73.

4. Blomberg J, Benachenhou F, Blikstad V, Sperber G, Mayer J. Classification and nomenclature of endogenous retroviral sequences (ERVs): problems and recommendations. Gene. 2008;448:115-23.

5. Vargiu L, Rodriguez-Tomé P, Sperber GO, Cadeddu M, Grandi N, Blikstad $\checkmark$, Tramontano E, Blomberg J. Classification and characterization of human endogenous retroviruses; mosaic forms are common. Retrovirology. 2016;13:7.

6. Cohen M, Larsson E. Human endogenous retroviruses. BioEssays. 1988:9:191-6.

7. Jern P, Sperber GO, Blomberg J. Use of endogenous retroviral sequences (ERVs) and structural markers for retroviral phylogenetic inference and taxonomy. Retrovirology. 2005;2:50.

8. Pavlícek A, Paces J, Elleder D. Processed pseudogenes of human endogenous retroviruses generated by LINEs: their integration, stability, and distribution. Genome Res. 2002;12:391-9.

9. Mager DL, Goodchild NL. Homologous recombination between the LTRs of a human retrovirus-like element causes a 5-kb deletion in two siblings. Am J Hum Genet. 1989;45:848-54.

10. Villesen $P$, Aagaard $L$, Wiuf $C$, Pedersen FS. Identification of endogenous retroviral reading frames in the human genome. Retrovirology. 2004;1:32.

11. Blond $\mathrm{J}$, Besème $F$, Duret $L$, Bouton $\mathrm{O}$, Bedin $\mathrm{F}$, Perron $\mathrm{H}$, Mandrand $\mathrm{B}$, Mallet F. Molecular characterization and placental expression of HERVW, a new human endogenous retrovirus family. J Virol. 1999;73:1175-85.

12. Mi S, Lee X, Li X, Veldman GM, Finnerty H, Racie L, Lavallie E, Tang X, Edouard P, Howes S Jr, Keith JC, Mccoy JM. Syncytin is a captive retroviral envelope protein involved. Nature. 2000;403(February):785-9.

13. Blond JL, Lavillette D, Cheynet V, Bouton O, Oriol G, Chapel-Fernandes $\mathrm{S}$, Mandrand B, Mallet F, Cosset FL. An envelope glycoprotein of the human endogenous retrovirus HERV-W is expressed in the human placenta and fuses cells expressing the type $D$ mammalian retrovirus receptor. JVirol. 2000;74:3321-9. 
14. Mangeney M, Renard M, Schlecht-Louf G, Bouallaga I, Heidmann O, Letzelter C, Richaud A, Ducos B, Heidmann T. Placental syncytins: Genetic disjunction between the fusogenic and immunosuppressive activity of retroviral envelope proteins. Proc Natl Acad Sci U S A. 2007;104:20534-9.

15. Christensen T. Association of human endogenous retroviruses with multiple sclerosis and possible interactions with herpes viruses. Rev Med Virol. 2005;1 5:179-211.

16. Perron $\mathrm{H}$, Perin JP, Rieger F, Alliel PM. Particle-associated retroviral RNA and tandem RGH/HERV-W copies on human chromosome 7q: possible components of a "chain-reaction" triggered by infectious agents in multiple sclerosis? J Neurovirol. 2000;6(Suppl 2):S67-75.

17. Perron H, Lazarini F, Ruprecht K, Péchoux-Longin C, Seilhean D, Sazdovitch V, Créange A, Battail-Poirot N, Sibaï G, Santoro L, Jolivet M, Darlix J-L, Rieckmann P, Arzberger T, Hauw J-J, Lassmann H. Human endogenous retrovirus (HERV)-W ENV and GAG proteins: physiological expression in human brain and pathophysiological modulation in multiple sclerosis lesions. J Neurovirol. 2005;11:23-33.

18. Brudek T, Christensen T, Aagaard L, Petersen T, Hansen HJ, Møller-Larsen A. B cells and monocytes from patients with active multiple sclerosis exhibit increased surface expression of both HERV-H Env and HERV-W Env, accompanied by increased seroreactivity. Retrovirology. 2009;6:104.

19. García-Montojo M, de la Hera B, Varadé J, de la Encarnación A, Camacho I, Domínguez-Mozo M, Arias-Leal A, García-Martínez A, Casanova I, Izquierdo G, Lucas M, Fedetz M, Alcina A, Arroyo R, Matesanz F, Urcelay E, Alvarez-Lafuente R. HERV-W polymorphism in chromosome X is associated with multiple sclerosis risk and with differential expression of MSRV. Retrovirology. 2014;11:2.

20. Schmitt K, Richter C, Backes C, Meese E, Ruprecht K, Mayer J. Comprehensive analysis of human endogenous retrovirus group HERV-W locus transcription in multiple sclerosis brain lesions by high-throughput amplicon sequencing. J Virol. 2013;87:13837-52.

21. Hon GM, Erasmus RT, Matsha T. Multiple sclerosis-associated retrovirus and related human endogenous retrovirus-W in patients with multiple sclerosis: a literature review. J Neuroimmunol. 2013;263:8-12.

22. Karlsson H, Bachmann S, Schröder J, McArthur J, Torrey EF, Yolken $\mathrm{RH}$. Retroviral RNA identified in the cerebrospinal fluids and brains of individuals with schizophrenia. Proc Natl Acad Sci U S A. 2001;98:4634-9.

23. Perron H, Mekaoui L, Bernard C, Veas F, Stefas I, Leboyer M. Endogenous retrovirus type W GAG and envelope protein antigenemia in serum of schizophrenic patients. Biol Psychiatry. 2008:64:1019-23.

24. Frank O, Giehl M, Zheng C, Hehlmann R, Leib-Mosch C, Seifarth W. Human endogenous retrovirus expression profiles in samples from brains of patients with schizophrenia and bipolar disorders. J Virol. 2005:79:10890-901.

25. Perron H, Hamdani N, Faucard R, Lajnef M, Jamain S, Daban-Huard C, Sarrazin S, LeGuen E, Houenou J, Delavest M, Moins-Teisserenc H, MoinsTeiserenc H, Bengoufa D, Yolken R, Madeira A, Garcia-Montojo M, Gehin N, Burgelin I, Ollagnier G, Bernard C, Dumaine A, Henrion A, Gombert A, Le Dudal K, Charron D, Krishnamoorthy R, Tamouza R, Leboyer M. Molecular characteristics of human endogenous retrovirus type-W in schizophrenia and bipolar disorder. Transl Psychiatry. 2012;2:e201 .

26. Bendiksen S, Martinez-Zubiavrra I, Tümmler C, Knutsen G, Elvenes J, Olsen E, Olsen R, Moens U. Human endogenous retrovirus W activity in cartilage of osteoarthritis patients. Biomed Res Int. 2014;2014:1-14.

27. Maliniemi P, Vincendeau M, Mayer J, Frank O, Hahtola S, Karenko L, Carlsson E, Mallet F, Seifarth W, Leib-Mösch C, Ranki A. Expression of human endogenous retrovirus-w including syncytin-1 in cutaneous T-cell lymphoma. PLoS ONE. 2013;8:e76281.

28. Antony JM, Deslauriers AM, Bhat RK, Ellestad KK, Power C. Human endogenous retroviruses and multiple sclerosis: innocent bystanders or disease determinants? Biochim Biophys Acta. 2011;1812:162-76.

29. Magiorkinis G, Belshaw R, Katzourakis A. "There and back again”: revisiting the pathophysiological roles of human endogenous retroviruses in the post-genomic era. Philos Trans R Soc Lond B Biol Sci. 2013;368:20120504.

30. Voisset C, Bouton O, Bedin F, Duret L, Mandrand B, Mallet F, ParanhosBaccala G. Chromosomal distribution and coding capacity of the human endogenous retrovirus HERV-W family. AIDS Res Hum Retrovir. 2000;16:731-40.
31. Costas J. Characterization of the intragenomic spread of the human endogenous retrovirus family HERV-W. Mol Biol Evol. 2002;19:526-33.

32. Perron H, Germi R, Bernard C, Garcia-Montojo M, Deluen C, Farinelli L, Faucard R, Veas F, Stefas I, Fabriek BO, Van-Horssen J, Van-der-Valk P, Gerdil C, Mancuso R, Saresella M, Clerici M, Marcel S, Creange A, Cavaretta R, Caputo D, Arru G, Morand P, Lang AB, Sotgiu S, Ruprecht K, Rieckmann P, Villoslada P, Chofflon M, Boucraut J, Pelletier J, et al. Human endogenous retrovirus type $W$ envelope expression in blood and brain cells provides new insights into multiple sclerosis disease. Mult Scler. 2012;18:1721-36.

33. Varmus HE. Form and function of retroviral proviruses. Science. 1982;216:812-20.

34. Kim H-S. Genomic impact, chromosomal distribution and transcriptional regulation of HERV elements. Mol Cells. 2012;33:539-44.

35. Hedges DJ, Deininger PL. Inviting instability: transposable elements, double-strand breaks, and the maintenance of genome integrity. Mutat Res. 2007;616:46-59.

36. Khodosevich K, Lebedev Y, Sverdlov E. Endogenous retroviruses and human evolution. Comp Funct Genom. 2002;3:494-8.

37. Jern $P$, Coffin JM. Effects of retroviruses on host genome function. Annu Rev Genet. 2008;42:709-32.

38. Schön U, Diem O, Leitner L, Günzburg WH, Mager DL, Salmons B, Leib-Mösch C. Human endogenous retroviral long terminal repeat sequences as cell type-specific promoters in retroviral vectors. J Virol. 2009;83:12643-50.

39. Kowalski PE, Freeman JD, Mager DL. Intergenic splicing between a HERV-H endogenous retrovirus and two adjacent human genes. Genomics. 1999;57:371-9.

40. Medstrand P, Landry JR, Mager DL. Long terminal repeats are used as alternative promoters for the endothelin B receptor and apolipoprotein C-I genes in humans. J Biol Chem. 2001;276:1896-903.

41. Dunn CA, Medstrand P, Mager DL. An endogenous retroviral long terminal repeat is the dominant promoter for human beta 1,3-galactosyltransferase 5 in the colon. Proc Natl Acad Sci U S A. 2003;100:12841-6.

42. Jordan IK, Rogozin IB, Glazko GV, Koonin EV. Origin of a substantial fraction of human regulatory sequences from transposable elements. Trends Genet. 2003;19:68-72.

43. van de Lagemaat LN, Landry J-R, Mager DL, Medstrand P. Transposable elements in mammals promote regulatory variation and diversification of genes with specialized functions. Trends Genet. 2003;19:530-6.

44. Dunn CA, van de Lagemaat LN, Baillie GJ, Mager DL. Endogenous retrovirus long terminal repeats as ready-to-use mobile promoters: the case of primate beta3GAL-T5. Gene. 2005;364:2-12.

45. Medstrand P, van de Lagemaat LN, Dunn CA, Landry J-R, Svenback D, Mager DL. Impact of transposable elements on the evolution of mammalian gene regulation. Cytogenet Genome Res. 2005;1 10:342-52.

46. Sin HS, Huh JW, Kim DS, Kang DW, Min DS, Kim TH, Ha HS, Kim HH, Lee SY, Kim HS. Transcriptional control of the HERV-H LTR element of the GSDML gene in human tissues and cancer cells. Arch Virol. 2006;151:1985-94.

47. Piriyapongsa J, Polavarapu N, Borodovsky M, McDonald J. Exonization of the LTR transposable elements in human genome. BMC Genom. 2007;8:291.

48. Conley AB, Piriyapongsa J, Jordan IK. Retroviral promoters in the human genome. Bioinformatics. 2008;24:1563-7.

49. Isbel L, Whitelaw E. Endogenous retroviruses in mammals: an emerging picture of how ERVs modify expression of adjacent genes. BioEssays. 2012:34:734-8.

50. Sperber GO, Airola T, Jern P, Blomberg J. Automated recognition of retroviral sequences in genomic data-RetroTector. Nucleic Acids Res. 2007:35:4964-76.

51. Kent WJ. BLAT—the BLAST-like alignment tool. Genome Res. 2002;12:656-64.

52. Jurka J, Kapitonov W, Pavlicek A, Klonowski P, Kohany O, Walichiewicz J. Repbase update, a database of eukaryotic repetitive elements. Cytogenet Genome Res. 2005;110:462-7.

53. Subramanian RP, Wildschutte JH, Russo C, Coffin JM. Identification, characterization, and comparative genomic distribution of the HERV-K (HML-2) group of human endogenous retroviruses. SM1. Retrovirology. 2011;8:90. 
54. Pavlíček A, Pačes J, Zíka R, Hejnar J. Length distribution of long interspersed nucleotide elements (LINEs) and processed pseudogenes of human endogenous retroviruses: implications for retrotransposition and pseudogene detection. Gene. 2002;300:189-94.

55. Dangel AW, Mendoza AR, Menachery CD, Baker BJ, Daniel CM, Carroll MC, Wu LC, Yu CY. The dichotomous size variation of human complement C4 genes is mediated by a novel family of endogenous retroviruses, which also establishes species-specific genomic patterns among old world primates. Immunogenetics. 1994:40:425-36.

56. Lebedev YB, Belonovitch OS, Zybrova NV, Khil PP, Kurdyukov SG, Vinogradova TV, Hunsmann G, Sverdlov ED. Differences in HERV-K LTR insertions in orthologous loci of humans and great apes. Gene. 2000;247:265-77.

57. Kim HS, Takenaka O, Crow TJ. Isolation and phylogeny of endogenous retrovirus sequences belonging to the HERV-W family in primates. J Gen Virol. 1999;80:2613-9.

58. Perelman $P$, Johnson WE, Roos $C$, Seuánez HN, Horvath JE, Moreira MAM, Kessing B, Pontius J, Roelke M, Rumpler Y, Schneider MPC, Silva A, O'Brien SJ, Pecon-Slattery J. A molecular phylogeny of living primates. PLoS Genet. 2011;7:1-17.

59. Blomberg J, Benachenhou F, Blikstad V, Sperber G, Mayer J. Classification and nomenclature of endogenous retroviral sequences (ERVs): problems and recommendations. Gene. 2009;448:115-23.

60. Bowzard JB, Bennett RP, Krishna NK, Ernst SM, Rein A, Wills JW. Importance of basic residues in the nucleocapsid sequence for retrovirus Gag assembly and complementation rescue. J Virol. 1998;72:9034-44.

61. Malik HS, Eickbush TH. Modular evolution of the integrase domain in the Ty3/Gypsy class of LTR retrotransposons. J Virol. 1999;73:5186-90.

62. Singleton TL, Levin HL. A long terminal repeat retrotransposon of fission yeast has strong preferences for specific sites of insertion. Eukaryot Cell. 2002; $1: 44-55$

63. Jern P, Sperber GO, Ahlsén G, Blomberg J. Sequence variability, gene structure, and expression of full-length human endogenous retrovirus H. J Virol. 2005;79:6325-37.

64. Zsíros J, Jebbink MF, Lukashov VV, Voûte PA, Berkhout B. Biased nucleotide composition of the genome of HERV-K related endogenous retroviruses and its evolutionary implications. J Mol Evol. 1999;48:102-11.

65. Mangeat B, Turelli P, Caron G, Friedli M, Perrin L, Trono D. Broad antiretroviral defence by human APOBEC3G through lethal editing of nascent reverse transcripts. Nature. 2003:424:99-103.

66. Chiu Y-L, Greene WC. The APOBEC3 cytidine deaminases: an innate defensive network opposing exogenous retroviruses and endogenous retroelements. Annu Rev Immunol. 2008:26:317-53.

67. Chiu Y-L, Witkowska HE, Hall SC, Santiago M, Soros VB, Esnault C, Heidmann T, Greene WC. High-molecular-mass APOBEC3G complexes restrict Alu retrotransposition. Proc Natl Acad Sci U S A. 2006;103:15588-93.

68. van de Lagemaat LN, Medstrand P, Mager DL. Multiple effects govern endogenous retrovirus survival patterns in human gene introns. Genome Biol. 2006;7:R86.

69. Medstrand P, Van De Lagemaat LN, Mager DL. Retroelement distributions in the human genome: variations associated with age and proximity to genes. Genome Res. 2002;12:1483-95.

70. Li F, Nellåker C, Yolken RH, Karlsson H. A systematic evaluation of expression of HERV-W elements; influence of genomic context, viral structure and orientation. BMC Genom. 2011;12:22.

71. Hadjiargyrou M, Delihas N. The intertwining of transposable elements and non-coding RNAs. Int J Mol Sci. 2013;14:13307-28.

72. Venter JC, Adams MD, Myers EW, Li PW, Mural RJ, Sutton GG, Smith HO, Yandell M, Evans CA, Holt RA, Gocayne JD, Amanatides P, Ballew RM, Huson DH, Wortman JR, Zhang Q, Kodira CD, Zheng XH, Chen L, Skupski M, Subramanian G, Thomas PD, Zhang J, Gabor Miklos GL, Nelson C, Broder S, Clark AG, Nadeau J, McKusick VA, Zinder N, et al. The sequence of the human genome. Science. 2001;291:1304-51.

73. Gimenez J, Mallet F. ERVWE1 (endogenous retroviral family W, Env(C7), member 1). Atlas Genet Cytogenet Oncol Haematol. 2008;12:134-48.

74. de Parseval N, Lazar V, Casella J-F, Benit L, Heidmann T. Survey of human genes of retroviral origin: identification and transcriptome of the genes with coding capacity for complete envelope proteins. J Virol. 2003;77:10414-22.
75. Roebke C, Wahl S, Laufer G, Stadelmann C, Sauter M, Mueller-Lantzsch N, Mayer J, Ruprecht K. An N-terminally truncated envelope protein encoded by a human endogenous retrovirus $\mathrm{W}$ locus on chromosome Xq22.3. Retrovirology. 2010;7:69.

76. Cheynet V, Ruggieri A, Oriol G, Blond J-L, Boson B, Vachot L, Verrier B, Cosset F-L, Mallet F. Synthesis, assembly, and processing of the Env ERVWE1/syncytin human endogenous retroviral envelope. J Virol. 2005;79:5585-93.

77. Bonnaud B, Bouton O, Oriol G, Cheynet V, Duret L, Mallet F. Evidence of selection on the domesticated ERVWE1 env retroviral element involved in placentation. Mol Biol Evol. 2004;21:1895-901.

78. Schiavetti F, Thonnard J, Colau D, Boon T, Coulie PG. A human endogenous retroviral sequence encoding an antigen recognized on melanoma by cytolytic T lymphocytes. Cancer Res. 2002;62:5510-6.

79. Perron C, Geny A, Laurent C, Mouriquand J, Pellat J, Perret J, Seigneurin J. Leptomeningeal cell line from multiple sclerosis with reverse transcriptase activity and viral particles. Res Virol. 1989;140:551-61.

80. Perron H, Lalande B, Gratacap B, Laurent A, Genoulaz O, Geny C, Mallaret $\mathrm{M}$, Schuller E, Stoebner P, Seigneurin J. Isolation of retrovirus from patients with multiple sclerosis. Lancet. 1991;337:862-3.

81. Komurian-Pradel F, Paranhos-Baccala G, Bedin F, Ounanian-Paraz A, Sodoyer M, Ott C, Rajoharison A, Garcia E, Mallet F, Mandrand B, Perron H. Molecular cloning and characterization of MSRV-related sequences associated with retrovirus-like particles. Virology. 1999;260:1-9.

82. Perron H, Garson JA, Bedin F, Beseme F, Paranhos-Baccala G, KomurianPradel F, Mallet F, Tuke PW, Voisset C, Blond JL, Lalande B, Seigneurin JM, Mandrand B. Molecular identification of a novel retrovirus repeatedly isolated from patients with multiple sclerosis. The Collaborative Research Group on Multiple Sclerosis. Proc Natl Acad Sci U S A. 1997:94:7583-8.

83. Garcia-Montojo M, Dominguez-Mozo M, Arias-Leal A, Garcia-Martinez Á, de las Heras V, Casanova I, Faucard R, Gehin N, Madeira A, Arroyo R, Curtin F, Alvarez-Lafuente R, Perron H. The DNA copy number of human endogenous retrovirus-W (MSRV-Type) is increased in multiple sclerosis patients and is influenced by gender and disease severity. PLoS One 2013;8:e53623.

84. Mameli G, Astone V, Arru G, Marconi S, Lovato L, Serra C, Sotgiu S, Bonetti B, Dolei A. Brains and peripheral blood mononuclear cells of multiple sclerosis (MS) patients hyperexpress MS-associated retrovirus/ HERV-W endogenous retrovirus, but not human herpesvirus 6. J Gen Virol. 2007;88(Pt 1):264-74.

85. Mameli G, Poddighe L, Astone V, Delogu G, Arru G, Sotgiu S, Serra C, Dolei A. Novel reliable real-time PCR for differential detection of MSRVenv and syncytin-1 in RNA and DNA from patients with multiple sclerosis. J Virol Methods. 2009;161:98-106.

86. Dolei A, Perron H. The multiple sclerosis-associated retrovirus and its HERV-W endogenous family: a biological interface between virology, genetics, and immunology in human physiology and disease. J Neurovirol. 2009:15:4-13.

87. Blomberg J, Ushameckis D, Jern P. Evolutionary aspects of human endogenous retroviral sequences (HERVs) and disease. In: Sverdlov ED, editor. Retroviruses and primate genomes evolution. Austin: Landes Bioscience; 2000. pp. 204-238.

88. Voisset C, Weiss RA, Griffiths DJ. Human RNA "rumor"viruses: the search for novel human retroviruses in chronic disease. Microbiol Mol Biol Rev. 2008;72:157-96 (table of contents).

89. Laufer G, Mayer J, Mueller BF, Mueller-Lantzsch N, Ruprecht K. Analysis of transcribed human endogenous retrovirus $W$ env loci clarifies the origin of multiple sclerosis-associated retrovirus env sequences. Retrovirology. 2009;6:37.

90. Flockerzi A, Maydt J, Frank O, Ruggieri A, Maldener E, Seifarth W, Medstrand P, Lengauer T, Meyerhans A, Leib-Mösch C, Meese E, Mayer $\mathrm{J}$. Expression pattern analysis of transcribed HERV sequences is complicated by ex vivo recombination. Retrovirology. 2007:4:39.

91. Deb-Rinker P, Klempan TA, O'Reilly RL, Torrey EF, Singh SM. Molecular characterization of a MSRV-like sequence identified by RDA from monozygotic twin pairs discordant for schizophrenia. Genomics. 1999;61:133-44.

92. Blaise S, de Parseval N, Heidmann T. Functional characterization of two newly identified human endogenous retrovirus coding envelope genes. Retrovirology. 2005;2:19. 
93. Christensen T. HERVs in neuropathogenesis. J Neuroimmune Pharmacol. 2010;5:326-35.

94. Chance MR, Sagi I, Wirt MD, Frisbie SM, Scheuring E, Chen E, Bess JW $J \mathrm{~J}$, Henderson LE, Arthur LO, South TL, et al. Extended x-ray absorption fine structure studies of a retrovirus: equine infectious anemia virus cysteine arrays are coordinated to zinc. Proc Natl Acad Sci U S A. 1992;89:10041-5.

95. James Kent W, Sugnet CW, Furey TS, Roskin KM, Pringle TH, Zahler AM, Haussler D. The human genome browser at UCSC. Genome Res. 2002;12:996-1006.

96. Katoh K, Standley DM. MAFFT multiple sequence alignment software version 7: improvements in performance and usability. Mol Biol Evol. 2013;30:772-80.

97. Kearse M, Moir R, Wilson A, Stones-Havas S, Cheung M, Sturrock S, Buxton S, Cooper A, Markowitz S, Duran C, Thierer T, Ashton B, Meintjes P, Drummond A. Geneious basic: an integrated and extendable desktop software platform for the organization and analysis of sequence data. Bioinformatics. 2012;28:1647-9.

98. Tamura K, Stecher G, Peterson D, Filipski A, Kumar S. MEGA6: molecular evolutionary genetics analysis version 6.0. Mol Biol Evol. 2013;30:2725-9.

99. Crooks GE, Hon G, Chandonia JM, Brenner SE. WebLogo: a sequence logo generator. Genome Res. 2004;14:1188-90.

100. Jühling F, Mörl M, Hartmann RK, Sprinzl M, Stadler PF, Pütz J. tRNAdb 2009: compilation of tRNA sequences and tRNA genes. Nucleic Acids Res. 2009;37(Database issue):D159-62.

101. Harrow J, Frankish A, Gonzalez JM, Tapanari E, Diekhans M, Kokocinski F, Aken BL, Barrell D, Zadissa A, Searle S, Barnes I, Bignell A, Boychenko V, Hunt T, Kay M, Mukherjee G, Rajan J, Despacio-Reyes G, Saunders G, Steward C, Harte R, Lin M, Howald C, Tanzer A, Derrien T, Chrast J, Walters N, Balasubramanian S, Pei B, Tress M, et al. GENCODE: the reference human genome annotation for the ENCODE project. Genome Res. 2012;22:1760-74.
102. Hsu F, Kent WJ, Clawson H, Kuhn RM, Diekhans M, Haussler D. The UCSC known genes. Bioinformatics. 2006;22:1036-46.

103. Pruitt KD, Tatusova T, Maglott DR. NCBI Reference Sequence (RefSeq): a curated non-redundant sequence database of genomes, transcripts and proteins. Nucleic Acids Res. 2005;33(Database issue):D501-4.

104. Karolchik D, Barber GP, Casper J, Clawson H, Cline MS, Diekhans M, Dreszer TR, Fujita PA, Guruvadoo L, Haeussler M, Harte RA, Heitner S, Hinrichs AS, Learned K, Lee BT, Li CH, Raney BJ, Rhead B, Rosenbloom KR, Sloan CA, Speir ML, Zweig AS, Haussler D, Kuhn RM, Kent WJ. The UCSC Genome Browser database: 2014 update. Nucleic Acids Res. 2014:42(Database issue):D764-70

105. Gerstein MB, Kundaje A, Hariharan M, Landt SG, Yan K-K, Cheng C, Mu XJ, Khurana E, Rozowsky J, Alexander R, Min R, Alves P, Abyzov A, Addleman N, Bhardwaj N, Boyle AP, Cayting P, Charos A, Chen DZ, Cheng Y, Clarke D, Eastman C, Euskirchen G, Frietze S, Fu Y, Gertz J, Grubert F, Harmanci A, Jain P, Kasowski M, et al. Architecture of the human regulatory network derived from ENCODE data. Nature. 2012;489:91-100.

106. Wang J, Zhuang J, lyer S, Lin X, Whitfield TW, Greven MC, Pierce BG, Dong X, Kundaje A, Cheng Y, Rando OJ, Birney E, Myers RM, Noble WS, Snyder M, Weng Z. Sequence features and chromatin structure around the genomic regions bound by 119 human transcription factors. Genome Res. 2012;22:1798-812

107. Maydt J, Lengauer T. Recco: recombination analysis using cost optimization. Bioinformatics. 2006;22:1064-71.

\section{Submit your next manuscript to BioMed Central and we will help you at every step:}

- We accept pre-submission inquiries

- Our selector tool helps you to find the most relevant journal

- We provide round the clock customer support

- Convenient online submission

- Thorough peer review

- Inclusion in PubMed and all major indexing services

- Maximum visibility for your research

Submit your manuscript at www.biomedcentral.com/submit
( Biomed Central 